\title{
Generalized Whitehead Spaces with Few Cells
}

\author{
Dedicated to the memory of Professor J. Frank Adams
}

By

Norio IWASE*, Akira Kono** and Mamoru MimurA***

\section{\$®. Imerroduction}

A topological space $E$ is called a generalized Whitehead space (a GW-space, for short) if every generalized Whitehead product on $E$ is trivial. As is clearly seen, this notion is a stronger notion of a Whitehead space. Thus a GW-space is a simple space.

The following three are well known:

(0.1). E is a GW-space if and only if the loop addition of $\Omega E$ is homotopy commutative, i.e., $\mu \circ T \simeq \mu$ where $\mu$ is the loop addition and $T: X \times Y \rightarrow Y \times X$ is a switching map.

(0.2). E is a GW-space if and only if, for a space $W$, the homotopy set $[\Sigma W$, $E] \cong[W, \Omega E]$ is naturally an abelian group with respect to $W$.

(0.3). E is a GW-space if and only if for given maps $f: \Sigma X \rightarrow E$ and $g: \Sigma Y \rightarrow E$ there is an 'axial' map $H: \Sigma X \times \Sigma Y \rightarrow E$ with axes $(f, g)$.

Here we must designate a loop structure (a classifying space) of a loop space, when we say something about the homotopy commutativity, because there exists a space with two different loop structures: One is homotopy commutative but the other is not.

As is well known, the loop addition of the loop space of an $\mathrm{H}$-space is always homotopy commutative. Thus an $\mathrm{H}$-space is a $\mathrm{GW}$-space by (0.1). In other words, the notion of a GW-space is a weaker notion of an $\mathrm{H}$-space. For a suspended space, however, the two notions are equivalent. In particular, $S^{n}$ is a GW-space (at 2) if and only if $n=1,3$ or 7, by [Ad1]. Kachi has studied in [K] GW-spaces with two or three cells (other than the base point 0 -cell). He showed

This research was partially supported by Grant-in-Aid for Encouragement of Young Scientists A-02740039 from The Ministry of Education, Science and Culture.

Communicated by M. Kashiwara, September 18, 1991.

1991 Mathematics Subject Classifications: Primary 55Q15; Secondary 55P35, 55S25.

* Department of Mathematics, Okayama University, Okayama 700, Japan.

*** Department of Mathematics, Kyoto University, Kyoto 606, Japan.

决小 Department of Mathematics, Okayama University, Okayama 700, Japan. 
that there are no GW-spaces with two cells unless the space is contractible and restricted the possible type of a GW-space which is a total space of a spherical bundle over a sphere.

In this paper, we consider a three cell $\mathrm{CW}$ complex $E$ whose cells are in dimensions $0, q, n$ and $m$ with $0<q \leq n \leq m$, for example, the total space of a spherical bundle (or fibration) over a sphere which is studied in $[\mathrm{K}]$. We call such a complex a complex of type $(q, n, m)$. The purpose of this paper is to show

Theorem 1. If a complex E of type $(q, n, m)$ is a $G W$-space (at 2), then $E$ has the homotopy type of either a sphere of dimension 1,3 or 7, or a Poincaré complex of type $(q, n, q+n)$ where $\{q, n\} \subseteq\{1,3,7\}$ or $(q, n)=(1,2),(2,4)$, $(3,4),(3,5)$ or $(3,7)$. In the latter case, E has the homotopy type (at 2) of one of the following spaces (See $[H-R]$ and $[Z]$ for further details on $E_{k \omega}$ ).

$$
\begin{array}{ll}
S^{q} \times S^{n} & \text { for }\{q, n\} \subseteq\{1,3,7\}, \\
L^{3}(p, \ell) & \text { for }(q, n)=(1,2), \\
C P(3) & \text { for }(q, n)=(2,4), \\
S^{7} & \text { for }(q, n)=(3,4), \\
S U(3) & \text { for }(q, n)=(3,5), \\
E_{k \omega} & \text { for }(q, n)=(3,7)
\end{array}
$$

where $p \geq 1$, and $\ell$ is a unit of a group ring $Z \pi /\left(1+\tau+\ldots+\tau^{p-1}\right), \pi=$ $\left\langle\tau \mid \tau^{p}=1\right\rangle \cong Z / p Z$ and $k \neq 2 \bmod 4$.

Remark. (1) Since $\pi_{2}\left(S^{1} \cup_{p_{11}} e^{2}\right) \cong Z \pi /\left(1+\tau+\ldots+\tau^{p-1}\right) \quad(\pi=$ $\left.\pi_{1}\left(S^{1} \cup_{p_{1}} e^{2}\right)=Z / p Z \tau\right)$, $\ell$ determines a 3 -dimensional (general) lens space $L^{3}(p, \ell)=S^{1} \cup_{p_{11}} e^{2} \cup_{\ell} e^{3} \cdot L^{3}(p, \ell)$ is an $\mathrm{H}$-space if and only if $p=1$ or 2 . In each case, $L^{3}(p, \ell)$ is homotopy equivalent to $S^{3}$ or $R P^{3}$, respectively. A standard lens space $L^{3}(p, \tau)$ is a GW-space (see Appendix). Moreover it is a Gottlieb space ([I-Y]).

(2) $C P(3)$ is a well-known example which is a Whitehead space but not an H-space. Moreover it is a GW-space (see Appendix) but not a Gottlieb space.

(3) The manifold $E_{k \omega}$ is determined by $k \in Z / 12 Z$. In particular, $E_{0}=\mathbb{S}^{3} \times$ $S^{7}$ and $E_{ \pm \omega} \cong S p(2)$. It is known that $E_{k \omega}$ is an $\mathrm{H}$-space if and only if $k \neq 2 \bmod$ 4.

(4) A T-space in the sense of Aguade [Ag] is a GW-space and also a Gottlieb space. But we do not know the converse.

Let us propose the following

Conjecture 1. Every connected finite complex $G W$-space is a Poincaré complex. 
Conjecture 2. The rational cohomology of a connected finite complex GWspace is a tensor product of monogenic polynomial algebrae truncated at height greater than 2 and exterior algebrae on odd dimensional generators.

Conjecture 3. If $E$ is a connected finite $G W$-space such that $H^{\prime}(E ; Z)$ has no even dimensional generators, then $E$ is an H-space.

This paper is organized as follows. In $\S 1$, we study a space whose mod 2 cohomology is a truncated polynomial algebra of height 3 on two generators. In $\S 2$, we study a GW-space whose rational cohomology is a polynomial algebra on one generator truncated at height 4 . In $\S \S 3-5$, we study a GW-space whose integral cohomology is an exterior algebra on two generators. In the last section, $\S 6$, we prove the main theorem.

Throughout the paper, $G$ stands for $\Omega E$ whose loop addition is denoted by $\mu$. The abbreviations $H^{+}(X)$ and $K^{+}(X)$ will be used for $H^{k}\left(X ; Z_{(2)}\right)$ and $K^{+}(X$; $\left.Z_{(2)}\right)$, respectively. $\widetilde{H}^{+}$and $\widetilde{K}^{+}$denote the augmentation ideals. $P H^{+}(X, R)$ is the submodule of primitive elements and $Q H^{4}(X ; R)$ is the quotient module of indecomposables for a coefficient ring $R . R\{a, b, c, \ldots\}$ means that it is an $R$-module with generators $a, b, c, \ldots$

The first and second authors thank the Department of Mathematics of the University of Aberdeen for its hospitality. The authors thank Hideyuki Kachi and Yutaka Hemmi for suggestions on removing a technical condition in an earlier version of this paper and for discussions which helped them to get their ideas in order. The first author thanks the Professor Mimura and Hisami Iwase for translating and typing the first draft of the manuscript. This work was completed during the first and second authors' stay in Aberdeen.

\section{$\S 1$. A Stable GW-Space}

Suppose that there is a space $X$ satisfying

$$
H^{+}(X ; Z / 2 Z) \cong Z / 2^{[3]}\left[v_{q+1}, v_{n+1}\right] \text { with } q \leq n
$$

where the right hand side is the polynomial algebra truncated at height 3 with 2 generators $v_{q+1}$ and $v_{n+1}$ of dimension $q+1$ and $n+1$, respectively.

Hence $A=H^{\prime}(X ; Z / 2 Z)$ is a truncated polynomial algebra over the mod 2 Steenrod algebra $A(2)$. Then from Theorem 2.1 of [Th1] it follows that $q=2^{r}-1$ and $n=2^{r}+2^{s}-1(r-1 \geq s \geq 0)$ or $n=2^{t}-1(t \geq r)$. Again from Theorem 1.4 of [Th1] it follows that

$$
Q A^{i+j} \subseteq \operatorname{Im} S q^{J} \cap \operatorname{Ker} S q^{j} \text { if }\left(\begin{array}{c}
i-1 \\
j
\end{array}\right) \equiv 1 \bmod 2
$$

where $Q A^{+}$indicates the quotient module of indecomposables. 
Furthermore if one replaces $P_{2} E$ with our $X$ in the argument given in $\S 4$ of [Th2] and the result [Th2, 4.5] due to Browder with (1.2) in the above which does not suppose the existence of an $\mathbb{H}$-structure, one can obtain

$$
q=1,3,7 \text { or } 15 \text { and if } q=15 \text { then } X \text { has 2-torsion. }
$$

If $X$ has 2-torsion in its homology, then $n$ is even and $n=q+1$ and hence $v_{n+1}=S q^{1} v_{q+1}$. In particular, if $q=15$, then $n=16$ and $S q^{1} v_{16}=v_{17}$.

If $X$ has no 2-torsion in its homology, then one can define John Hubbuck's operations as follows: We have

$$
\begin{aligned}
& H^{4}(X) \cong Z_{(2)}^{[3]}\left[\bar{v}_{(q+1) / 2}, \bar{v}_{(n+1) / 2}\right], \\
& K^{\prime}(X) \cong Z_{(2)}^{[3]}\left[w_{(q+1) / 2}, w_{(n+1) / 2}\right] .
\end{aligned}
$$

Hence there is a ring isomorphism $J: H^{4}(X) \rightarrow K^{4}(X)$ given by

$$
J\left(\bar{v}_{i}\right)=w_{l}, \text { for } i=(q+1) / 2 \text { and }(n+1) / 2 .
$$

Now the Adams operation $\psi^{h}$ decomposes through Hubbuck operations $R_{J}^{h}(k)$ (see [Hu] for details) for an element $x_{n} \in H^{n}(X)$, as follows:

$$
J^{-1} \psi^{k} J\left(x_{n}\right)=\sum_{h=0}^{\infty} \frac{k^{n}}{2^{h}} R_{J}^{h}(k)\left(x_{n}\right)
$$

where $R_{J}^{h}(k)\left(x_{n}\right)$ increases dimension by $h$. The multiplicativity of Adams operations is expressed by using Hubbuck operations in the following Cartan formula:

$$
R_{J}^{h}(k)\left(v \cdot v^{\prime}\right)=\sum_{I^{+}=h} R_{J}^{\prime}(k)(v) \cdot R_{J}^{J}(k)\left(v^{\prime}\right) .
$$

Set $R^{h}=\frac{i}{2^{h}} R_{J}^{h}(3)$ and $P^{h}=R_{J}^{h}(2)$ so that the reduction $\bmod 2$ of $P^{h}$ is $S q^{2 h}$. The relation $\psi^{3} \psi^{2}=\psi^{2} \psi^{3}$ of Adams operations is expressed by using the Hubbuck operations as follows:

$$
\left(3^{n}-1\right) P^{n}+\sum_{t=1}^{n} 3^{n-i} 2^{i} R^{\prime} P^{n-1}=\sum_{t=1}^{n} 2^{2 i} P^{n-t} R^{\prime}
$$

Furthermore, the relation $\psi^{2}\left(x_{n}\right) \equiv x_{n}^{2} \bmod 2$ is interpreted as

$$
\begin{array}{ll}
P^{n+J}\left(x_{n}\right) \equiv 0 \bmod 2^{i+1} & \text { and } \\
P^{n}\left(x_{n}\right) \equiv x_{n}^{2} \bmod 2 & \text { in } H^{+}(X) .
\end{array}
$$

Note that the above formula is independent of the choice of the splitting $J$. Following (1.3), we check the cases $q=15,7,3$ and 1 , one by one.

Consider the case $q=15$. By (1.3) one has $n=16$ and $S q^{1} v_{16}=v_{17} . \mathbb{B y}(1.2)$ one has $v_{17} \in \operatorname{Im~} S q^{8}$, since $\left({ }^{9-1}\right) \equiv 1 \bmod 2$, but it contradicts $H^{9}(X ; Z / 2 Z)=0$. Thus $q \neq 15$.

Consider the case $q=7$ and $n=7+2^{s}$ with $s \leq 2$. If $s=0$, then $S q^{1} v_{8}=v_{9}$. 
By (1.2) $v_{9} \in \operatorname{Im} S q^{4}$, since $\left({ }^{5-1}\right) \equiv 1 \bmod 2$, but it contradicts $H^{5}(X ; Z / 2 Z)=0$. If $s=1$, then $n=9$ and $v_{10} \in \operatorname{Im} S q^{4}$, since $\left({ }^{6-1}\right) \equiv 1 \bmod 2$; but it contradicts $H^{6}(X ; Z / 2 Z)=0$. Thus $s=2$ and then $n=11$ and $v_{12} \in \operatorname{Im} S q^{4}$, since $\left({ }^{8-1}\right) \equiv 1$ $\bmod 2$. We have

$$
\begin{aligned}
H^{+}(X) \cong Z_{(2)}^{[3]}\left[\bar{v}_{4}, \bar{v}_{6}\right], \\
K^{+}(X) \cong Z_{(2)}^{[3]}\left[w_{4}, w_{6}\right],
\end{aligned}
$$

since the homology of $X$ is free of 2 torsion.

Thus $P^{\text {odd }}=R^{\text {odd }}=0$ and $P^{2} \bar{v}_{4} \equiv \bar{v}_{6} \bmod 2$. Then it follows from (1.4) that $R^{2} \equiv 2 P^{2} \bmod 4$ and $2 P^{6} R^{2} \equiv P^{2} P^{6}+2 R^{4} P^{4} \bmod 8$. Hence by (1.5) we obtain

$$
2 \bar{v}_{6}^{2} \equiv 2 P^{6}\left(\bar{v}_{6}\right) \equiv 2 P^{6} P^{2}\left(\bar{v}_{4}\right) \equiv P^{6} R^{2}\left(\bar{v}_{4}\right) \equiv R^{4} P^{4}\left(\bar{v}_{4}\right) \bmod 4 .
$$

Also from (1.5) it follows that $P^{4}\left(\bar{v}_{4}\right) \equiv \lambda \bar{v}_{4}^{2} \bmod 4$ for some odd integer $\lambda$. Hence by the equation $R^{2} \equiv 2 P^{2} \bmod 4$ with the Cartan formula, one obtains

$$
0 \not 2 \bar{v}_{6}^{2} \not \equiv \lambda R^{4}\left(\bar{v}_{4}^{2}\right) \equiv 2 \lambda \bar{v}_{4} R^{4}\left(\bar{v}_{4}\right) \quad \bmod 4 .
$$

It is a contradiction, since the right hand side does not contribute $2 \bar{v}_{6}^{2}$.

Thus $n \neq 7+2^{s}$ with $s \leq 2$.

Consider the case $q=7$ and $n=2^{t}-1$ with $t \geq 3$. If $t=3$, then $(q, n)=(7,7)$. If $t=4$, then $n=15$. We have

$$
\begin{aligned}
& H^{+}(X) \cong Z_{(2)}^{[3]}\left[\bar{v}_{4}, \bar{v}_{8}\right], \\
& K^{+}(X) \cong Z_{(2)}^{[3]}\left[w_{4}, w_{8}\right] .
\end{aligned}
$$

Thus $P^{\text {odd }}=P^{2 \cdot o d d}=0$. Then by (1.4) one obtains that

$$
2 P^{8} \equiv P^{4} P^{4} \bmod 4 \text { in } H^{4}(X) \text {. }
$$

By (1.5), one has that

$$
\begin{aligned}
& P^{4}\left(\bar{v}_{8}\right)=\alpha \bar{v}_{4} \bar{v}_{8} \\
& P^{8}\left(\bar{v}_{8}\right) \equiv \bar{v}_{8}^{2} \quad \bmod 2 \\
& P^{4}\left(\bar{v}_{4}\right) \equiv \bar{v}_{4}^{2} \quad \bmod 2
\end{aligned}
$$

and hence

$$
P^{4}\left(\bar{v}_{4}\right)=\lambda \bar{v}_{4}^{2}+2 \beta \bar{v}_{8},
$$

for some $\alpha, \beta$ and $\lambda \in Z_{(2)}$, where $\lambda \equiv 1 \bmod 2$.

Then from (1.6), it follows that

$$
\begin{aligned}
2 \bar{v}_{8}^{2} & \equiv 2 P^{8}\left(\bar{v}_{8}\right) \equiv P^{4} P^{4}\left(\bar{v}_{8}\right) \equiv \alpha P^{4}\left(\bar{v}_{4} \bar{v}_{8}\right) \quad \bmod 4 \\
& \equiv \alpha P^{4}\left(\bar{v}_{4}\right) \bar{v}_{8} \equiv 2 \alpha \beta \bar{v}_{8}^{2} \quad \bmod 4 .
\end{aligned}
$$

Thus $\alpha \beta \equiv 1 \bmod 2$. By using (1.5), however, it follows from (1.6) that 


$$
\begin{aligned}
0 & \equiv 2 P^{8}\left(\bar{v}_{4}\right) \equiv P^{4} P^{4}\left(\bar{v}_{4}\right) \equiv P^{4}\left(\lambda \bar{v}_{4}^{2}+2 \beta \bar{v}_{8}\right) \quad \bmod 4 \\
& \equiv 2 \lambda \bar{v}_{4} P^{4}\left(\bar{v}_{4}\right)+2 \beta P^{4}\left(\bar{v}_{8}\right) \equiv 2 \beta P^{4}\left(\bar{v}_{8}\right) \equiv 2 \alpha \beta \bar{v}_{4} \bar{v}_{8} \quad \bmod 4,
\end{aligned}
$$

which contradicts $\alpha \beta \equiv 1 \bmod 2$. Hence $t \neq 4$. If $t \geq 5$, we have

$$
H^{+}(X ; Z / 2 Z) \cong Z / 2^{[3]}\left[\bar{v}_{4}, \bar{v}_{2^{t-1}}\right] \text {. }
$$

Then from the main result of [Ad1], it follows that

$$
S q^{2^{t}} \equiv \sum_{i=0}^{t-1} S q^{2^{t}} \Psi_{i}
$$

modulo the total indeterminacy which is in the image of $S q^{i}$ with $2^{t}>i>0$. Now the formula gives a contradiction. In fact, the left hand side gives $S q^{2^{i}} v_{2^{i}} \neq \equiv$ mod 2 while the right hand side and the total indeterminacy are trivial, since

$$
H^{2^{i+1}-2^{i}}(X)=0 \text { for } i \leq t-1 \text {. }
$$

It is a contradiction.

Thus $(q, n)=(7,7)$, provided that $q=7$.

Consider the case $q=3$ and $n=3+2^{s}$ with $s \leq 1$. If $s=0$, then $n=4$ and $S q^{1} v_{4}=v_{5}$. We have $v_{5} \in \operatorname{Im} S q^{2}$ by (1.2), since $\left({ }^{3-1}\right) \equiv 1 \bmod 2$. This contradicts $H^{2}(X ; Z / 2 Z)=0$. Hence $s=1$ and then $n=5$ and $(q, n)=(3,5)$. Moreover we have $v_{6} \in \operatorname{Im} S q^{2}$ by $(1.2)$, since $\left({ }^{4-1}\right) \equiv 1 \bmod 2$.

Consider the case $q=3$ and $n=2^{t}-1$ with $t \geq 2$. If $t=2$, then $(q, n)=(3,3)$. If $t=3$, then $(q, n)=(3,7)$. If $t \geq 4$, then we will be led to a contradiction as in the case when $q=7$ and $n=2^{t}-1$ with $t \geq 5$.

Thus $(q, n)=(3,3),(3,5)$ or $(3,7)$, provided that $q=3$.

Consider the case $q=1$ and $n=1+2^{s}$ with $s \leq 0$. We have $s=0$ and hence $(q, n)=(1,2)$. Moreover by $(1.3), S q^{1} v_{2}=v_{3}$.

Consider the case $q=1$ and $n=2^{t}-1$ with $t \geq 1$. If $t=1$, then $(q, n)=(1,1)$. If $t=2$, then $(q, n)=(1,3)$. If $t=3$, then $(q, n)=(1,7)$. If $t \geq 4$, then we will be led to a contradiction as in the case when $q=7$ and $n=2^{t}-1$ with $t \geq 5$.

Thus $(q, n)=(1,1),(1,2),(1,3)$ or $(1,7)$, provided that $q=1$.

Therefore we have shown

Proposition 1.7. If there is a space $X$ such that

$$
H^{+}(X ; Z / 2 Z) \cong Z / 2^{[3]}\left[v_{q+1}, v_{n+1}\right]
$$

with $q \leq n$, then $\{q, n\} \subseteq\{1,3,7\}$ or $(q, n)=(1,2)$ or $(3,5)$. Moreover if $(q, n)=(1,2)$, then $S q^{1} v_{2}=v_{3}$; if $(q, n)=(3,5)$, then $S q^{2} v_{4}=v_{6}$.

To apply this, we introduce the following notion.

Definition 1.8. Let $E$ be a complex of type $(q, n, m) . E$ is said to be stable if $n<2 q$. 
We have

Corollary 1.9. Let $E$ be a Poincaré complex of type $(q, n, q+n)$. If $E$ is a stable $G W$-space (at 2), then $\{q, n\} \subseteq\{1,3,7\}$ or $(q, n)=(3,4)$ or $(3,5)$. In case $(q, n)=(3,4), E$ has the homotopy type of $S^{7}($ at 2$)$.

Proof. Let $\alpha$ be the attaching map of the $n$-cell of $E$ and let us write $E=S^{q} \cup_{\alpha} e^{n} \cup e^{q+n}$. By the hypothesis, $q>1$ or $\alpha=0$. Let $Q$ be the subspace $S^{q} \cup_{\alpha} e^{n}$ of $E$. Then, from the hypothesis, it follows that $Q$ is desuspendable and the mod 2 cohomology of $E$ is an exterior algebra except the case when $n=q+1$ and $\alpha=k \iota_{q}, k$ odd.

(Case 1: The mod 2 cohomology of $E$ is an exterior algebra). There exists an axial map $\mu$ : $Q \times Q \rightarrow E$ with axes $(j, j)$ where $j$ is the inclusion $Q \hookrightarrow E$. Let $Q(2)$ be the mapping cone of the Hopf construction of $\mu$. From a direct computation using [Th3], we obtain that the mod 2 cohomology of $Q(2)$ is the polynomial algebra truncated at height 3 on the generators in dimensions $q+1$ and $n+1$. Hence by Proposition 1.7 we obtain that $\{q, n\} \subset\{1,3,7\}$ or $(q, n)=(3,5)$.

(Case 2: $n=q+1$ and $\alpha=k \iota_{q}, k$ odd). $E$ has the homotopy type of a $(2 q+1)$-sphere at 2 . Hence by Adams' theorem [Ad1], $q=1$ or 3 . Thus $(q, n)=$ $(3,4)$ and $E$ has the homotopy type at 2 of $S^{7}$.

In case $(q, n)=(3,4)$ with an (integral) $\mathrm{GW}$-space structure on $E$, we get moreover that $k= \pm 1$. Assume that there is an odd prime $p$ such that $k=0$ $\bmod p$. Then the $\bmod p$ cohomology of $E$ is again an exterior algebra. Hence a similar construction of $Q(2)$ can be performed and one obtains that there exists an element of dimension 5 in its $\bmod p$ cohomology whose square is non-zero. It is a contradiction, since a square of any odd dimensional element of mod $p$ cohomology must be 0 when $p$ odd. Thus $k= \pm 1$ and $E$ has the homotopy type of $S^{7}$. This implies the corollary.

\section{§2. A GW-Space whose Cohomology is a Truncated Polynomial Algebra}

Let $E$ be a Poincaré complex of type $(q, 2 q, 3 q)$ such that $H^{+}(E ; Q) \cong$ $Q\left[x_{q}\right] /\left(x_{q}^{4}\right)$. So we have

$$
E=S^{q} \cup_{\alpha} e^{2 q} \cup e^{3 q}, \alpha \in \pi_{2 q-1}\left(S^{q}\right) .
$$

In this section, we will show

Proposition 2.1. If, further, $E$ is a $G W$-space (at 2), then $q=2$ and $H^{\dagger}\left(E ; Z_{(2)}\right) \cong Z_{(2)}\left[x_{2}\right] /\left(x_{2}^{4}\right)$.

The remainder of this section is devoted to proving the proposition.

By the assumption on the rational cohomology ring of $E, q$ is even $\geq 2$. 
Since $E$ is a Poincaré complex, we have the following isomorphism of algebrae.

$$
H^{+}\left(E ; Z_{(2)}\right) \cong Z_{(2)}\left\{x_{q}, x_{2 q}, x_{3 q}\right\},
$$

where $x_{q}^{2}=a x_{2 q}$ and $x_{q} x_{2 q}=x_{3 q}$ with $0 \neq a \in Z_{(2)}$.

Since $E$ is a GW-space, the Whitehead product of the inclusion $i: S^{q} \longrightarrow E$ vanishes, and hence $i_{+}\left[\iota_{q}, \iota_{q}\right]=0$ where $\iota_{q} \in \pi_{q}\left(S^{q}\right)$ is the class of the identity. Let us denote by $\widehat{i}: F \rightarrow S^{q}$ the homotopy fibre of $i$. Then there is a map $f$ : $S^{2 q-1} \rightarrow F$ such that $\hat{i} \circ f \simeq\left[\iota_{q}, \iota_{q}\right]$. One obtains

$$
F \simeq{ }_{2} S^{2 q-1} \cup(\text { higher dimensional cells) }
$$

so that $\left.\hat{i}\right|_{s^{2} q-1}=\alpha$. One may compress $f$ to the $(2 q-1)$-dimensional skeleton $S^{2 q-1}$ of $F$, one has $\left[\iota_{q}, \iota_{q}\right]=\alpha \circ f$, where $f=\lambda \iota_{2 q-1}: S^{2 q-1} \rightarrow S^{2 q-1}$ with $\lambda \in Z$ :

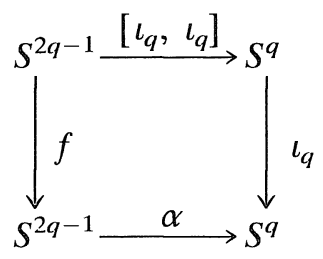

Then it follows that $\left[\iota_{q}, \iota_{q}\right]=\alpha \circ f=\lambda \alpha$. Taking the Hopf invariants of the both hand sides, one has $2=\lambda H(\alpha)$, whence $a=H(\alpha)= \pm 1$ or \pm 2 .

If $H(\alpha)= \pm 1$, then $q=2,4$ or 8 by [Ad1] and we obtain that $\left[\iota_{q}, \iota_{q}\right]$ is divisible by 2 . According to [To], this holds only when $q=2$ and then we have $H^{4}(E) \cong Z_{(2)}\left[x_{2}\right] /\left(x_{2}^{4}\right)$. Thus the following lemma implies Proposition 2.1:

Lemma 2.2. $H(\alpha)= \pm 1$ and hence $q=2,4$ or 8 .

The remainder of this section is devoted to prove the lemma.

Suppose $H(\alpha)= \pm 2$ so that $a= \pm 2, \alpha= \pm\left[\iota_{q}, \iota_{q}\right]$ and $\Sigma \alpha=0$. We will show that this assumption leads us to a contradiction. Now the $2 q$-skeleton of $G$ has the following cell decomposition:

$$
G^{[2 q]} \simeq_{2} S^{q-1} \cup_{\left[\iota_{q-1}, \iota_{q-1}\right]} e^{2 q-2} \cup e^{2 q-1} .
$$

Thus putting $Q=\Sigma\left(G^{[2 q]}\right)$, we have

$$
Q \simeq_{2}\left(S^{q} \vee S^{2 q-1}\right) \cup_{\bar{\alpha}} e^{2 q},
$$

where $\bar{\alpha}$ is in $\pi_{2 q-1}\left(S^{q} \vee S^{2 q-1}\right)$.

Let $l$ denotes the composite map of the canonical inclusion $Q \rightarrow \Sigma G$ and the evaluation $\lambda_{1}: \Sigma G=\Sigma \Omega E \rightarrow E$. To proceed, we need to show the following

Proposition 2.3. $\bar{\alpha}$ corresponds to $\left(\alpha, \pm 2 \iota_{2 q-1}\right)$ under the isomorphism $\pi_{2 q-1}\left(S^{q} \vee S^{2 q-1}\right) \cong \pi_{2 q-1}\left(S^{q}\right) \oplus \pi_{2 q-1}\left(S^{2 q-1}\right)$. 
Proof. By calculating the cohomology Serre spectral sequence associated with the path fibration $G \hookrightarrow P E \rightarrow E$, one obtains

$$
\begin{aligned}
& H^{q-1}(G) \cong Z_{(2)}, \\
& H^{q-1+j}(G)=0, \text { for } 1 \leq j \leq q-1, \\
& H^{2 q-1}(G) \cong Z / 2 Z
\end{aligned}
$$

Hence the composite map $p_{2} \circ \bar{\alpha}$ is homotopic to $\pm 2 \iota_{2 q-1}$, where $p_{t}$ indicates the projection to the $t$-th factor. Moreover $\ell$ induces the following commutative diagram for some integer $\lambda$ :

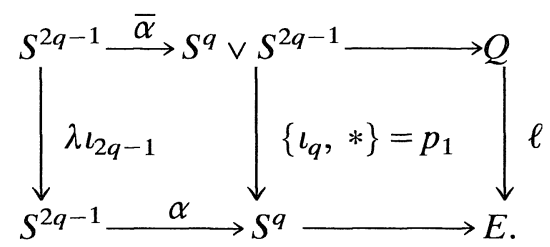

Here both the $q-1$ and the $2 q-1$ dimensional generators in $H^{+}(G)$ are transgressive and therefore $\ell$ induces a surjection of cohomology groups in dimensions $\leq 2 q$. Hence $\lambda=1$ and $p_{1} \circ \bar{\alpha}$ is homotopic to $\alpha$.

This implies Proposition 2.3.

By Proposition 2.3 one obtains that $l^{+}: H^{j}(E ; Z / 2 Z) \rightarrow H^{j}(Q ; Z / 2 Z)$ is an isomorphism for $j=q$ and $2 q$. So one may assume that $l^{+} x_{j}=y_{j}$ for $j=q$ and $2 q$, and that

$$
H^{4}(Q ; Z / 2 Z) \cong Z / 2 Z\left\{y_{q}, y_{2 q-1}, y_{2 q}\right\} \text {. }
$$

Let us recall that $Q$ is a suspended space and $E$ is a $\mathrm{GW}$-space. Hence by $(0.3)$ there exists an axial map

$$
\mu: Q \times Q \rightarrow E
$$

with axes $(l, l)$. So the Hopf construction of $\mu$ gives rise to a map

$$
H(\mu): \Sigma Q \wedge Q \simeq Q * Q \rightarrow \Sigma E
$$

so that

$$
\begin{aligned}
H(\mu)^{+}\left(\Sigma^{*} x_{q}\right) & =0, \\
H(\mu)^{+}\left(\Sigma^{+} x_{2 q}\right) & =\Sigma^{+} y_{q} \otimes y_{q}, \\
H(\mu)^{+}\left(\Sigma^{+} x_{3 q}\right) & =\Sigma^{+} y_{q} \otimes y_{2 q}+\Sigma^{*} y_{2 q} \otimes y_{q} .
\end{aligned}
$$

One can see that $\Sigma Q$ satisfies

$$
\Sigma Q \simeq_{2}\left(S^{q+1} \vee S^{2 q}\right) \cup_{\Sigma \bar{\alpha}} e^{2 q+1} .
$$

By combining Proposition 2.3 with $\Sigma \alpha=0$, one obtains that $\Sigma \bar{\alpha}$ corresponds 
to $\left(0, \pm 2 \iota_{2 q}\right)$ under the isomorphism $\pi_{2 q}\left(S^{q+1} \vee S^{2 q}\right) \cong \pi_{2 q}\left(S^{q+1}\right) \oplus \pi_{2 q}\left(S^{2 q}\right)$. Hence we obtain

$$
\Sigma Q \simeq_{2} \Sigma S^{q} \vee \Sigma M^{2 q},
$$

where $M^{2 q}=S^{2 q-1} \cup_{ \pm 2 \iota} e^{2 q}$. Thus we obtain

$$
\Sigma Q \wedge Q \simeq_{2} \Sigma\left(S^{q} \vee M^{2 q}\right) \wedge\left(S^{q} \vee M^{2 q}\right),
$$

which contains $\Sigma\left(M^{2 q} \wedge M^{2 q}\right)$. We denote by $\bar{H}(\mu)$ the restriction of $H(\mu)$ to the subcomplex $\Sigma\left(M^{2 q} \wedge M^{2 q}\right)$ and by $Q(2)$ the mapping cone of $\bar{H}(\mu)$. Then we have an exact sequence associated with it:

$$
\cdots \rightarrow \widetilde{H}^{+}-1\left(\Sigma\left(M^{2 q} \wedge M^{2 q}\right) ; Z / 2 Z\right) \stackrel{\delta}{\rightarrow} \widetilde{H}^{+}(Q(2) ; Z / 2 Z) \rightarrow \widetilde{H}^{+}(\Sigma E ; Z / 2 Z) \rightarrow \cdots
$$

For dimensional reasons, the sequence splits and we have

$$
\begin{aligned}
\widetilde{H}^{+}(Q(2) ; Z / 2 Z) & \cong Z / 2\left\{v_{q+1}, v_{2 q+1}, v_{3 q+1}\right\} \oplus \operatorname{Im} \delta, \\
\operatorname{Im} \delta & \cong \widetilde{H}^{*}\left(\Sigma\left(M^{2 q} \wedge M^{2 q}\right) ; Z / 2 Z\right) \\
& \cong Z / 2\left\{y_{2 q-1} \otimes y_{2 q-1}, y_{2 q-1} \otimes y_{2 q}, y_{2 q} \otimes y_{2 q-1}, y_{2 q} \otimes y_{2 q}\right\} .
\end{aligned}
$$

Then from [Th3] it follows that

$$
v_{2 q+1}^{2}=\delta \Sigma^{+}\left(y_{2 q} \otimes y_{2 q}\right) \neq 0
$$

and hence $0 \neq S q^{2 q+1} v_{2 q+1}$. Let us recall the Adem relation

$$
S q^{q} S q^{q+1}=S q^{2 q+1}+\left(\begin{array}{c}
q-1 \\
q-2
\end{array}\right) S q^{2 q} S q^{1}+\ldots+\left(\begin{array}{c}
\frac{q}{2} \\
0
\end{array}\right) S q^{3 q / 2+1} S q^{q / 2}
$$

for $q$ even. For $j$ with $1 \leq j \leq q / 2$, we have $\operatorname{deg} S q^{j} v_{2 q+1}=2 q+j+1<3 q+1<4 q$. Thus we obtain, for dimensional reasons,

$$
S q^{j} v_{2 q+1}=0 \text { for } 1 \leq j \leq q / 2 \text {. }
$$

Hence $S q^{q+1} v_{2 q+1} \neq 0$. The Adem relation $S q^{q+1}=S q^{1} S q^{q}$ ( $q$ even) implies that $S q^{q} v_{2 q+1} \neq 0$ and therefore $S q^{q} v_{2 q+1}=v_{3 q+1}$. Hence $S q^{1} v_{3 q+1} \neq 0$ where $\operatorname{deg} S q^{1} v_{3 q+1}=3 q+2 \leq 4 q$. Thus $3 q+2=4 q$ and hence $q=2$.

Even when $q=2$, one has

$$
S q^{1} v_{3 q+1}=\delta \Sigma^{+}\left(y_{2 q-1} \otimes y_{2 q-1}\right)
$$

and hence

$$
\begin{aligned}
0 & =S q^{1} S q^{1} v_{3 q+1} \\
& =\delta \Sigma^{*} S q^{1}\left(y_{2 q-1} \otimes y_{2 q-1}\right) \\
& =\delta \Sigma^{+}\left(y_{2 q} \otimes y_{2 q-1}+y_{2 q-1} \otimes y_{2 q}\right) \neq 0,
\end{aligned}
$$

which is a contradiction. This implies that $\Sigma \alpha \neq 0$. Thus $H(\alpha)= \pm 1$ and hence $q=2,4$ or 8 . 
This implies Lemma 2.2 and it completes the proof of Proposition 2.1.

\section{§3. A GW-Space whose Cohomology is an Exterior Algebra}

Throughout the section let $E$ be a (non-stable) Poincaré complex of type $(q, n, q+n)$. Let us assume that $E$ is a GW-space at 2 (or at $p$ for $p$ odd) such that

$$
H^{4}(E ; R)=\wedge\left(x_{q}, x_{n}\right), 1 \leq q<n
$$

where the coefficient ring $R$ is $Z_{(2)}$ (or $Z / p$, respectively).

We adopt the abbreviation $H^{+}(E)$ for $H^{+}(E ; R)$ if it does not cause a confusion.

If $q=1$ and $R=Z_{(2)}$, then the universal covering space $\widetilde{E}$ of $E$ has the homotopy type (at 2) of $S^{n}$, which inherits the GW-space structure. Let us recall that a sphere is a GW-space (at 2) if and only if it is an H-space. Hence $n=3$ or 7 .

We will prove that both $q$ and $n$ are odd integers, when $q>1$.

Let $q>1$. First we show

Proposition 3.1. $q$ is odd.

Consider the cohomology Serre spectral sequence with $R$ coefficient associated with the path fibration $G \hookrightarrow P E \rightarrow E$. Since the element $x_{q} \in H^{q}(E)$ is in the image of the transgression, we have $0 \neq \sigma^{\dagger} x_{q} \in H^{q-1}(G) \cong R$, where $\sigma^{+}$: $H^{+}(E) \rightarrow H^{+-1}(G)$ is the cohomology suspension. So $u_{q-1}=\sigma^{+} x_{q}$ is transgressive, and hence is primitive. Thus the element $\Sigma^{+} u_{q-1} \in H^{q}(\Sigma G)$ is extendable to the projective plane $P^{2} G$ and the extension is given by the image of $x_{q}$ under the induced map of the composite map

$$
\lambda_{2}: P^{2} G \hookrightarrow P^{\infty} G \simeq E
$$

since $\sigma^{+} x_{q}$ is represented by a loop map whose delooping is given by $x_{q}$. Hence we obtain

$$
\bar{x}_{q}^{2}=0 \quad \text { in } H^{+}\left(P^{2} G\right),
$$

where the element $\bar{x}_{q}^{2}$ is given by $\bar{x}_{q}^{2}= \pm \delta_{2} \Sigma^{+}\left(u_{q-1} \otimes u_{q-1}\right)$ and $\delta_{2}$ is the connecting homomorphism of Mayer-Vietoris exact sequence given in [Th2]. So it follows from the triviality of $\bar{x}_{q}^{2}$ that $u_{q-1} \otimes u_{q-1}$ is in the image of $\bar{\mu}^{+}=\mu^{+}-$ $p_{1}^{+}-p_{2}^{+}$:

$$
\bar{\mu}^{+}=\mu^{+}-p_{1}^{+}-p_{2}^{+}: \widetilde{H}^{+}(G) \rightarrow \widetilde{H}^{+}(G) \otimes \widetilde{H}^{+}(G) .
$$

So by $(0.1)$ we obtain that the element $u_{q-1} \otimes u_{q-1}$ is $T^{+}$-invariant where $T$ is the switching map. If $q$ is even, then $T^{+}\left(u_{q-1} \otimes u_{q-1}\right)=-u_{q-1} \otimes u_{q-1}$. Hence $u_{q-1} \otimes u_{q-1}$ is not $T^{+}$-invariant, since it is a generator of $\widetilde{H}^{2(q-1)}(G \wedge G) \cong$ $\widetilde{H}^{q-1}(G) \otimes \widetilde{H}^{q-1}(G)$ which has no 2 -torsion. Thus $q$ has to be odd and this 
implies the proposition.

Next we show

\section{Proposition 3.2. $n$ is odd.}

Suppose that $n$ is even. Then $n-1(\geq q-1)$ is odd and is not divisible by $q-1$, because $q-1$ is known to be even. Let us recall the following exact sequence for bicommutative biassociative Hopf algebra over $Z / p$ the prime field of characteristic $p$ :

$$
0 \rightarrow P\left(Z / p Z\left(\xi_{p} H^{+}(G ; Z / p Z)\right)\right) \rightarrow P H^{+}(G ; Z / p Z) \rightarrow Q H^{+}(G ; Z / p Z) .
$$

Then by the Serre spectral sequence associated with the fibration $G=\Omega E \rightarrow$ $P E \rightarrow E$, it follows that $u_{n-1}=\sigma^{4} x_{n}$ generates $H^{n-1}(G) \cong R$ and hence is primitive indecomposable. As in the proof of (3.1), the element $\Sigma^{+} u_{n-1}$ is extendable over $P^{2} G$. Denoting the extended element by $\bar{x}_{n}$, we have

$$
\bar{x}_{n}^{2}=0 \text { in } H^{+}\left(P^{2} G\right)
$$

since $\bar{x}_{n}=\lambda_{2}^{+}\left(x_{n}\right)$.

It means that the element $u_{n-1} \otimes u_{n-1}$ is in the image of $\bar{\mu}^{-1}$. On the other hand, $u_{n-1} \otimes u_{n-1}$ generates the direct summand $\widetilde{H}^{n-1}(G) \otimes \widetilde{H}^{n-1}(G) \cong R$ in $\widetilde{H}^{2 n-2}(G \wedge G)$, which cannot be in the image of $\bar{\mu}^{+}$. It implies that $u_{n-1} \otimes$ $u_{n-1} \notin \operatorname{Im} \bar{\mu}^{+}$. It is a contradiction. This implies that $n$ is odd and this implies the proposition.

Thus we have shown

Proposition 3.3. (1) Let $q=1<n$ and $R=Z_{(2)}$. If $E$ is a $G W$-space at 2, then $n=3$ or 7 . (2) Let $1<q<n$ and a ring $R$ be $Z_{(2)}$ (or $Z / p Z$ for $p$ odd). If $E$ is a GW-space at 2 (or at $p$, respectively) with $H^{\dagger}(E ; R)=\wedge\left(x_{q}, x_{n}\right)$, then both $q$ and $n$ are odd.

In the remainder of this section, assuming $q>1$ and $R=Z_{(2)}$, we study further on the dimensions $q$ and $n$ using the cohomology structure of $G$. We remark that $q+1<n$, since $q$ and $n$ are odd.

Now we choose an inclusion map $j: S^{q} \rightarrow E$ such that $j^{1} x_{q}$ is a generator of $H^{q}\left(S^{q}\right) \cong Z_{(2)}$. Recall that we do not assume the existence of a fibration $S^{q} \hookrightarrow$ $E \rightarrow S^{n}$. Let $F$ be the homotopy fibre of $j$. Thus $F \rightarrow S^{q} \rightarrow E$ and $\Omega S^{q} \hookrightarrow G \rightarrow F$ are Serre fibrations. Then by the Serre spectral sequence associated with $F \rightarrow$ $S^{q} \rightarrow E$ one sees

$$
H^{+}(F) \cong H^{\dagger}\left(\Omega S^{n}\right)
$$

which is concentrated in even dimensions. Hence the Serre spectral sequence associated with the fibration $\Omega S^{q} \hookrightarrow G \rightarrow F$ collapses and we obtain 


$$
H^{+}(G) \cong H^{+}\left(\Omega S^{q}\right) \otimes H^{+}\left(\Omega S^{n}\right) \text { as modules. }
$$

In particular

$$
H^{+}(G) \cong H^{+}\left(\Omega S^{q}\right) \text { for } *<n-1 .
$$

Here a system of ring generators of $H^{+}\left(\Omega S^{q}\right)$ is given by

$$
u_{q-1}=\gamma_{1} u_{q-1}, \gamma_{2} u_{q-1}, \ldots, \gamma_{j} u_{q-1}, \ldots,
$$

where $j \geq 1$ and $u_{q-1}=\sigma^{4} x_{q}$.

One obtains from (3.4) the following extension of bicommutative biassociative Hopf algebrae:

$$
Z_{(2)} \rightarrow H^{+}\left(\Omega S^{n}\right) \rightarrow H^{+}(G) \rightarrow H^{+}\left(\Omega S^{q}\right) \rightarrow Z_{(2)} .
$$

The following is a commutative diagram of exact sequences:

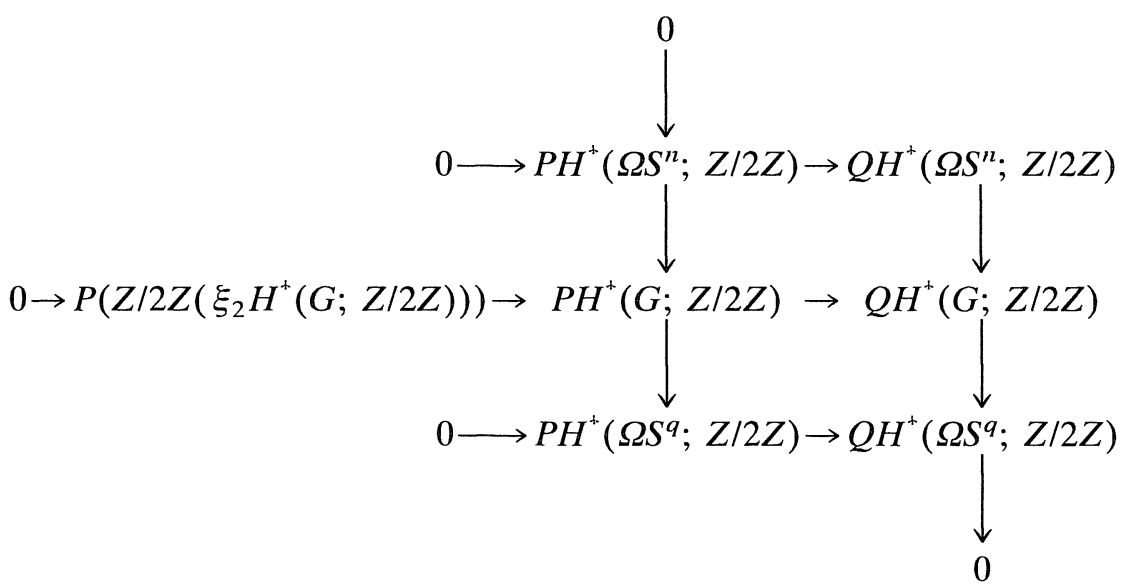

where $P H^{+}\left(\Omega S^{n} ; Z / 2 Z\right) \cong Z / 2 Z \tilde{u}_{n-1}$ (and $\dot{P} H^{+}\left(\Omega S^{q} ; Z / 2 Z\right) \cong Z / 2 Z \tilde{u}_{q-1}$, resp.) in which the element $\tilde{u}_{t-1}$ is the modulo 2 reduction of $u_{t-1}$ for $t=q$ and $n$.

Proposition 3.7. The first non-trivial relation in the algebra structure of $H^{+}(G)$ can occur in dimension $n-1$ when $n-1=2(q-1)$. The possible relation is

$$
\tilde{u}_{n-1}=\tilde{u}_{q-1}^{2} .
$$

Proof. It follows from $\left(3.4^{\prime}\right)$ and (3.5) that $\tilde{u}_{q-1}$ is primitive and hence $P H^{+}(G ; Z / 2 Z)$ is generated by $\tilde{u}_{n-1}$ and $\tilde{u}_{q-1}$. By (3.5), the first non-trivial relation in the algebra structure can occur in dimension $n-1$ only when $n-1=$ $2^{j}(q-1)$ for some integer $j>0$. Then the possible relation is 


$$
\tilde{u}_{n-1}=\left(\gamma_{2}, \tilde{u}_{q-1}\right)^{2} \text {. }
$$

Since $\tilde{u}_{q-1}$ is the only primitive element in $H^{+}\left(\Omega S^{q} ; Z / 2 Z\right)$, we obtain that $j$ must be 1 . This implies the proposition.

We show the following

Theorem 3.8. (i) $q \equiv 3 \bmod 4$,

(ii) If $n \equiv 1 \bmod 4$, then $\tilde{x}_{n}=S q^{2} \tilde{x}_{q}$ and $(q, n)=(3,5)$,

where $\tilde{x}_{t}$ is the modulo 2 reduction of $x_{t}$ for $t=q$ and $n$.

The remainder of this section is devoted to proving this theorem. First in the general situation, we will construct a space and compute its cohomology ring. The cell structure of the $n$-skeleton of $G$ is as follows:

$$
G^{[n]} \simeq_{2}\left(\Omega S^{q}\right)^{[n-1]} \cup e^{n-1} .
$$

Thus putting $Q=\Sigma\left(G^{[n]}\right)$, we have

$$
Q \simeq_{2}\left(\bigvee_{i=1}^{\left[\frac{n-1}{q-1}\right]} S^{i(q-1)+1}\right) \cup e^{n}
$$

The module $Q H^{*}(E)$ is mapped injectively into $H^{+}(Q)$ by the homomorphism induced from the composite map $\ell: Q \rightarrow E$ of the canonical inclusion $Q \subset \Sigma G$ and the evaluation $\lambda_{1}: \Sigma G \subset P^{\infty} G \simeq E$.

In fact, as was already seen, $P H^{*}(G) \cong Z_{(2)}\left\{u_{q-1}, u_{n-1}\right\}$ with $u_{i}$ transgressive, and $\ell^{*}$ gives rise to the cohomology suspension. Thus we obtain

$$
\operatorname{Im}(\Sigma \ell)^{*} \cong Z_{(2)}\left\{v_{q+1}, v_{n+1}\right\}
$$

which is a direct summand of $\widetilde{H}^{+}(\Sigma Q)$. Hence we have

$$
\widetilde{H}^{+}(\Sigma Q) \cong \operatorname{Im}(\Sigma \ell)^{+} \oplus D,
$$

where $D$ is the module generated by elements $\gamma_{i} u_{q-1}$ with $i \geq 2$. Since $Q$ is a suspension space, there exists an axial map

$$
\mu: Q \times Q \rightarrow E
$$

with axes $(\ell, \ell)$. So the Hopf construction of $\mu$ gives rise to a map

$$
H(\mu): \Sigma Q \wedge Q \simeq Q * Q \rightarrow \Sigma E .
$$

We denote by $Q(2)$ the mapping cone of $H(\mu)$, and then we have a cofibre sequence

$$
\Sigma E \stackrel{j}{\rightarrow} Q(2) \rightarrow \Sigma Q \wedge \Sigma Q .
$$

The elements $x_{q}, x_{n} \in \widetilde{H}^{+}(E)$ are primitive with respect to $\mu$ in the sense of Thomas [Th 3], since $\widetilde{H}^{\text {odd }}(Q \wedge Q)=0$. Hence we have 


$$
\begin{aligned}
& \tilde{\mu}^{+}\left(x_{i}\right)=0 \text { for } i=q \text { and } n, \\
& \tilde{\mu}^{+}\left(x_{q} x_{n}\right)=\ell^{+} x_{q} \otimes \ell^{+} x_{n}-\ell^{*} x_{n} \otimes \ell^{*} x_{q} .
\end{aligned}
$$

So the image of $j^{+}$induced by the inclusion $j: \Sigma E \hookrightarrow Q(2)$ are given by

$$
\operatorname{Im} j^{+} \cong Z_{(2)}\left\{\Sigma^{+} x_{q}, \Sigma^{+} x_{n}\right\} \text {. }
$$

Also the image and the kernel of the homomorphism $\delta$ induced from the collapsing map $Q(2) \rightarrow \Sigma Q \wedge \Sigma Q \cong \Sigma^{4}\left(G^{[n]} \wedge G^{[n]}\right)$ is given by

$$
\begin{aligned}
& \text { Ker } \delta \cong\left(\Sigma^{4}\right)^{+} Z_{(2)}\left\{u_{q-1} \otimes u_{n-1}-u_{n-1} \otimes u_{q-1}\right\}, \\
& \operatorname{Im} \delta \cong \delta\left(\Sigma^{4}\right)^{+} Z_{(2)}\left\{u_{i} \otimes u_{j} ; i, j=q-1 \text { or } n-1\right\} \oplus S_{2}
\end{aligned}
$$

where $S_{2} \cong \delta\left(D \otimes \widetilde{H}^{+}(\Sigma Q)\right) \oplus \delta\left(\widetilde{H}^{+}(\Sigma Q) \otimes D\right)$. Therefore by (3.9), we obtain the following short exact sequence:

$$
0 \rightarrow \operatorname{Im} \delta \rightarrow \widetilde{H}^{+}(Q(2)) \rightarrow Z_{(2)}\left\{\Sigma^{+} x_{q}, \Sigma^{+} x_{n}\right\} \rightarrow 0 .
$$

Thus denoting by $v_{i+1}$ the extension of $\Sigma^{+} x_{i}$ over $Q(2), i=q$ and $n$, we obtain the following ring isomorphisms by virtue of [Th3]:

$$
\begin{aligned}
& H^{+}(Q(2)) \cong Z_{(2)}^{[3]}\left[v_{q+1}, v_{n+1}\right] \oplus S_{2}, \\
& \widetilde{H}^{+}(Q(2)) \cdot S_{2}=0,
\end{aligned}
$$

where $v_{i+1} \cdot v_{j+1}=\delta\left(\Sigma^{4}\right)^{+}\left(u_{i-1} \otimes u_{j-1}\right)$.

We remark that these results are independent of the choice of $v_{q+1}$ and $v_{n+1}$.

Proposition 3.12. (1) $Q(2)$ has no torsion and hence $S q^{1} \widetilde{H}^{+}(Q(2) ; Z / 2 Z)=0$. (2) $\mathscr{A}(2)\left(Z / 2 Z\left\{\tilde{v}_{q+1}, \tilde{v}_{n+1}\right\}\right) \subset Z / 2 Z^{[3]}\left[\tilde{v}_{q+1}, \tilde{v}_{n+1}\right] \oplus\left(S_{2} \otimes Z / 2 Z\right)$, where $\tilde{v}_{t}$ is the modulo 2 reduction of $v_{t}$ for $t=q+1$ and $n+1$.

(3) $\theta(\delta \otimes Z / 2 Z)=(\delta \otimes Z / 2 Z) \theta$ for any $\theta \in \mathscr{A}(2)$, where $\delta: H^{4}\left(\Sigma^{2} Q \wedge Q\right) \rightarrow$ $H^{+}(Q(2))$.

The following two propositions imply Theorem 3.8 .

Proposition 3.13. If $n \equiv 1 \bmod 4$, then $\tilde{x}_{n}=S q^{2} \tilde{x}_{q}$ and $(q, n)=(3,5)$.

Proof. By (3.11), $H^{+}(Q(2) ; Z / 2 Z)$ has a direct summand $Z / 2 Z^{[3]}\left[\tilde{v}_{q+1}\right.$, $\left.\tilde{v}_{n+1}\right]$, where $\tilde{v}_{t}$ is the modulo 2 reduction of $v_{t}$ for $t=q+1$ and $n+1$. If $n=$ $4 k+1$ for some $k \geq 1$, we have

$$
0 \neq \tilde{v}_{n+1}^{2}=S q^{4 k+2} \tilde{v}_{n+1} .
$$

Since $S q^{4 k+2}=S q^{2} S q^{4 k}+S q^{1} S q^{4 k} S q^{1}$, one obtains that $\tilde{v}_{n+1}^{2} \in \operatorname{Im} S q^{2}$, since $S q^{1}=0$ on $H^{+}(Q(2) ; Z / 2 Z)$. Then it follows that $\tilde{v}_{n+1}^{2}=\delta\left(\Sigma^{4}\right)^{+}\left(\tilde{u}_{n-1} \otimes \tilde{u}_{n-1}\right) \in$ $S q^{2} \operatorname{Im} \delta$, where $\tilde{u}_{t}$ is the modulo 2 reduction of $u_{t}, t=q+1$ and $n+1$, for dimensional reasons. Hence one obtains that $\tilde{u}_{n-1} \otimes \tilde{u}_{n-1} \in \operatorname{Im} S q^{2}$ in 
$H^{*}\left(G^{[n]} \wedge G^{[n]} ; Z / 2 Z\right)$ modulo the kernel of $\delta \otimes Z / 2 Z$.

By (3.10), we have $Z / 2 Z\left\{\tilde{u}_{n-1} \otimes \tilde{u}_{n-1}\right\} \cap \operatorname{Ker} \delta=0$, which implies that $\tilde{u}_{n-1} \otimes \tilde{u}_{n-1} \in \operatorname{Im} S q^{2}$. Thus we obtain that $\tilde{u}_{n-1} \in \operatorname{Im} S q^{2}$ in $\widetilde{H}^{+}\left(G^{[n]} ; Z / 2 Z\right)$.

There are two cases: $\tilde{u}_{n-1}$ is indecomposable or not.

If $\tilde{u}_{n-1}$ is decomposable, one obtains $\tilde{u}_{n-1} \dot{\tilde{u}}_{q-1}^{2}$ by Proposition 3.7 , and hence $\tilde{u}_{q-1}^{2} \in \operatorname{Im} S q^{2}$. Then for dimensional reasons, then $\tilde{u}_{q-1}^{2}=S q^{2} \tilde{u}_{q-1}$ and hence $q-1=2$. This implies that $(q, n)=(3,5)$ and $\tilde{u}_{n-1}=S q^{2} \tilde{u}_{q-1}$, and hence $\tilde{x}_{n}=S q^{2} \tilde{x}_{q}$.

If $\tilde{u}_{n-1}$ is indecomposable, then there exists a non-negative integer $r \geq 0$ such that $S q^{2} \gamma_{2}, \tilde{u}_{q-1}=\tilde{u}_{n-1}$ with $2<2^{r}(q-1)$. Comparing the dimensions of both hand sides, one obtains $2+2^{r}(q-1)=n-1=4 m$, whence one has $r=0$, since $q-1$ is even by Proposition 3.3 (2). This implies that $S q^{2} \tilde{u}_{q-1}=\tilde{u}_{n-1} \neq 0$ and hence $n=q+2>4$ and $Q \simeq_{2} S^{q} \cup e^{n}$. Then the $\bmod 2$ cohomology of $Q(2)$ satisfies the condition given in $\$ 1$. Hence from Corollary 1.9 it follows that $(q, n)=(q, q+2)$ have to be $(3,5)$ which contradicts $2<2^{r}(q-1)$.

This implies the proposition.

Proposition 3.14. $q \equiv 3 \bmod 4$.

Proof. We consider $\widetilde{H}^{*}(Q(2) ; Z / 2 Z)$ which is given in the proof of the above proposition. We have $\tilde{v}_{q+1}^{2} \neq 0$ in $\widetilde{H}^{*}(Q(2) ; Z / 2 Z)$.

Assume that $q \equiv 1 \bmod 4$. Then one has $\tilde{v}_{q+1}^{2} \in \operatorname{Im} S q^{2}$. Also $\operatorname{deg} \tilde{v}_{q+1}^{2}-2=$ $2 q \equiv 2 \bmod 4$. If $n \equiv 1 \bmod 4$, then $q=3 \neq 1 \bmod 4$, which is a contradiction. So $n \equiv 3 \bmod 4$, whence $2 q \neq n+1$. Thus, one has that $\tilde{v}_{q+1}^{2} \in S q^{2} \operatorname{Im} \delta$. By a similar argument to that given in the proof of Proposition 3.13, we obtain that $\tilde{u}_{q-1} \otimes \tilde{u}_{q-1} \in \operatorname{Im} S q^{2}$ in $\widetilde{H}^{*}\left(G^{[n]} \wedge G^{[n]} ; Z / 2 Z\right)$. This implies that $\tilde{u}_{q-1} \otimes \tilde{u}_{q-1}=$ $S q^{2} \tilde{u}_{n-1}$ in $H^{+}\left(G^{[n]}\right)$, since $G^{[n]}$ is $(q-2)$ connected. Then by comparing dimensions, we have $2(q-1)=(n-1)+2$ and hence $n=2 q-3<2 q$. Hence from Corollary 1.9 it follows that $(q, n)=(q, 2 q-3)$ have to be $(3,5)$ which contradicts $q \equiv 1 \bmod 4$. This implies the proposition.

\section{§4. Non Stable GW-Spaces}

Let $E$ be a GW-space Poincaré complex of type $(q, n, q+n)$ such that $\widetilde{H}^{*}(E ; Z / 2 Z)=\wedge\left(x_{q}, x_{n}\right)$ with $1 \leq q<n . E$ has the homotopy type of $S^{q} \cup_{\alpha}$ $e^{n} \cup_{\beta} e^{n+q}$ where $\alpha \in \pi_{n-1}\left(S^{q}\right)$ and $\beta \in \pi_{n+q-1}\left(S^{q} \cup S^{n}\right)$.

Definition 4.1. $E \simeq S^{q} \cup_{\alpha} e^{n} \cup_{\beta} e^{q+n}$ is said to be non-stable if $2 q \leq n$. In other words, $\alpha$ is not in the stable range.

By Proposition 3.3 (2), we have that both $q$ and $n$ are odd integers. So $2 q<n$, if $E$ is non-stable. 
We will show

Theorem 4.2. If the above $E$ is a non-stable $G W$-space, then $(q, n)$ is one of the following: $(1,3),(1,7),(3,7),(3,11)$ or $(7,15)$.

The remainder of this section is devoted to proving Theorem 4.2.

Let $j: S^{q} \rightarrow E$ be the inclusion of the bottom sphere $S^{q}$. Consider the map $\{j, j\}: S^{q} \vee S^{q} \rightarrow E$. We have that the Whitehead product $[j, j]$ is homotopic to zero, as $E$ is a GW-space. Hence the map $\{j, j\}$ is extendable over $S^{q} \times S^{q} \rightarrow E$. By the assumption that $2 q<n$, the image of $\mu$ is compressible into $S^{q}$ so that $S^{q}$ is an H-space, whence $q=1,3$ or 7 by Adams' theorem [Ad1].

[The case $q=1$ ] The universal covering space $\widetilde{E}$ of $E$ is easily seen to be a GW-space having the same homotopy type as $S^{n}$, which then becomes an H-space. Again by the theorem of [Ad1], $n=1,3$ or 7 . Omitting the case $n=1$, we have $(q, n)=(1,3)$ or $(1,7)$.

[The case $q=3$ or 7] Put $\varepsilon=1$ or 3 according as $q=3$ or 7 , i.e. $\varepsilon=(q-1) / 2$. If $n \equiv 1 \bmod 4$, we obtain, by Theorem 3.8 , that $(q, n)=(3,5)$, which contradicts $n>2 q$. Hence $n \equiv 3 \bmod 4$. If the element $u_{n-1}=\sigma^{4} x_{n}$ in $P H^{n-1}(E ; Z / 2 Z)$ is decomposable in $H^{4}(G ; Z / 2 Z)$, then by the commutativity of 3.6 it is in the image of $\xi_{2}: P H^{4}(G ; Z / 2 Z) \rightarrow P H^{4}(G ; Z / 2 Z)$. It is impossible by the fact that $n-1 \equiv 2 \bmod 4$. Thus $u_{n-1}$ is indecomposable in $H^{+}(G ; Z / 2 Z)$.

Proposition 4.3. If $S q^{2} \neq 0$ on $H^{+}(G ; Z / 2 Z)$, then $n=2^{i+2} \varepsilon+3$ for some $i \geq 0$.

Proof. Put $u_{q-1}=\sigma^{+} x_{q}$ and $u_{n-1}=\sigma^{+} x_{n}$. Let $\omega \in H^{+}(G ; Z / 2 Z)$ be an element of the lowest dimension such that $S q^{2} \omega \neq 0$. Then $S q^{2} \omega$ is primitive, and so $S q^{2} \omega=u_{q-1}$ or $u_{n-1}$. It follows from $H^{q-3}(G ; Z / 2 Z)=0$, that $S q^{2} \omega=$ $u_{n-1}$. Thus $\omega$ is a generator of lower dimension than $n-1$, whence one can express it as $\omega=\gamma_{2^{i}+1} u_{q-1}$ for some $i \geq 0$ (, since $\gamma_{1} u_{q-1}=u_{q-1}$ is not mapped to $u_{n-1}$ by $\left.S q^{2}\right)$. Comparing the dimensions we have $2^{i+1}(q-1)+2=n-1$, and so $n=2^{i+1} \varepsilon+3$ for some $i \geq 0$.

This implies the proposition.

Proposition 4.4. If $S q^{2}=0$ on $H^{+}(G ; Z / 2 Z)$, then $S q^{2^{i}} H^{+}(G ; Z / 2 Z)=0$ for any $i \geq 0$.

Proof. Suppose $S q^{1}=\ldots=S q^{2^{t^{-1}}}=0$ and $S q^{2^{\prime}} \neq 0$ on $H^{*}(G ; Z / 2 Z)$. By assumption we have $j \geq 2$. As in the proof of Proposition 4.3, one can conclude that

$$
S q^{2^{\prime}} \gamma_{2^{i+1}} u_{q-1}=u_{n-1} \text { for some } i \geq 0
$$


since $\gamma_{1} u_{q-1}=u_{q-1}$ is not mapped to $u_{n-1}$ by any squaring operation from the fact that $2(q-1)<n-1$. Comparing the dimensions one has $2^{i+1}(q-1)+2^{j}=$ $n-1$; it gives $n-1 \equiv 0 \bmod 4$, since $j \geq 2$ and $q-1 \equiv 0 \bmod 2$. This contradicts $n \equiv 3 \bmod 4$.

This implies the proposition.

Corollary 4.5. If $u_{n-1} \in \operatorname{Im} S q^{2^{\prime}}$ in $H^{+}(G ; Z / 2 Z)$, then $j=1$.

We will discuss the two cases, whether $S q^{2}$ acts trivially or not, by using the methods given in $\$ 3$.

Theorem 4.6. If $S q^{2}=0$ on $H^{+}(G ; Z / 2 Z)$, then $(q, n)=(3,7)$.

Proof. It follows from Proposition 4.4 that every mod 2 Steenrod operation acts trivially on $H^{+}(G ; Z / 2 Z)$. Let $Q(2)$ be as in $\S 3$, then we have

$$
H^{+}\left(Q(2) ; Z_{(2)}\right) \cong Z_{(2)}^{[3]}\left[v_{q+1}, v_{n+1}\right] \oplus S_{2},
$$

To proceed, we need the following proposition, which is an immediate consequence of (3.10), (3.11), Proposition 3.12 and Proposition 4.4:

Proposition 4.7. If $v_{n+1}^{2} \in \operatorname{Im} S q^{t}$ in the algebra $H^{+}\left(Q(2) ; Z_{(2)}\right)$ for some $t>0$ and if $S q^{2}=0$ on $H^{*}(G ; Z / 2 Z)$, then $t \geq n+1$.

Now we will examine the decomposition of $S q^{2^{k+1}}(k \geq 0)$ through secondary operations on the space $X=Q(2)$, which is the main result in [Ad1]. If $n+1$ is not a power of 2 , then by the Adem relation one has

$$
0 \neq v_{n+1}^{2}=S q^{n+1}\left(v_{n+1}\right)=\sum_{i} a_{i} b_{i}\left(v_{n+1}\right), 0<\operatorname{deg} a_{i}<n+1
$$

which contradicts Proposition 4.7.

When $n=2^{k+4}-1, k \geq 0$, there holds

$$
0 \neq v_{n+1}^{2}=S q^{n+1}\left(v_{n+1}\right)=\sum_{i, j} a_{i j} \Phi_{i j}\left(v_{n+1}\right), 0<\operatorname{deg} a_{i j}<n+1
$$

modulo $a_{i j k} Q^{2 n+2-l}(i, j, k)(Q(2) ; Z / 2 Z)$ where $0<l(i, j, k)=\operatorname{deg} a_{i j k}<n+1$. Thus the element $v_{n+1}^{2}$ belongs to the image of a certain Steenrod operation $a$ with $0<\operatorname{deg} a<n+1$. This also contradicts Proposition 4.7. So, if $n+1=2^{k}$, then $k=0,1,2$ or 3 .

The equation $2 q=4 \varepsilon+1<n=2^{k}-1$ implies that $n=7$ if $q=3$ and that $n$ does not exist if $q=7$.

This completes the proof of Theorem 4.6. 
Theorem 4.8. If $S q^{2} \neq 0$ on $\widetilde{H}^{+}(G ; Z / 2 Z)$, then $(q, n)=(3,7),(3,11)$ or $(7,15)$.

Proof. It follows from Proposition 4.3 that $n=2^{i+2} \cdot \varepsilon+3$ for some $i \geq 0$.

If $i=0$, then $(q, n)=(3,7)$ or $(7,15)$.

We assume $i \geq 1$. Then $n+1=2^{i+2} \cdot \varepsilon+4 \equiv 4 \bmod 8$. So by the Adem relation we have

$$
\begin{aligned}
S q^{4} S q^{2^{1+2} \cdot \varepsilon} & =S q^{n+1}+S q^{2^{+2} \cdot \varepsilon+2} S q^{2}+S q^{2^{1+2} \cdot \varepsilon+3} S q^{1} \\
& =S q^{n+1}+S q^{2+2^{1+2} \cdot \varepsilon} S q^{2}+S q^{3} S q^{2^{1+2} \varepsilon} S q^{1} .
\end{aligned}
$$

Again by (3.10), (3.11) and Proposition 3.12, we obtain

$$
S q^{2} v_{n+1} \in \delta\left(\Sigma^{4}\right)^{+} \widetilde{H}^{+}\left(\Omega S^{q} \wedge \Omega S^{q}\right) \subseteq \delta\left(\Sigma^{4}\right)^{+} H^{+}(G \wedge G)
$$

since $\operatorname{deg} S q^{2} v_{n+1}=2+\operatorname{deg} v_{n+1}=4+\operatorname{deg} u_{n-1}\left(=4+2^{i+2} \cdot \varepsilon+2\right)$. Thus the following conditions are necessary for $S q^{2+2^{1+2} \cdot \varepsilon} S q^{2} v_{n+1}$ to contribute to $v_{n+1}^{2}=$ $\delta\left(\Sigma^{4}\right)^{+}\left(u_{n-1} \otimes u_{n-1}\right)$ : There are elements $\hat{u}_{i_{1}}$ and $\hat{u}_{i_{2}}$ of degree $i_{1}$ and $i_{2}$, respectively, such that

$$
\begin{gathered}
S q^{2} v_{n+1}=\delta \Sigma^{4}\left(\sum \hat{u}_{i_{1}} \otimes \hat{u}_{i_{2}}\right), \\
S q^{2+2^{2+2} \varepsilon\left(\Sigma \hat{u}_{i_{1}} \otimes \hat{u}_{i_{2}}\right)}=u_{n-1} \otimes u_{n-1}+\text { independent terms }
\end{gathered}
$$

modulo decomposables, where the summation ranges over the pairs $\left(i_{1}, i_{2}\right)$ with $i_{1}+i_{2}=\operatorname{deg} S q^{2} v_{n+1}-4=\operatorname{deg} u_{n-1}=2+2^{i+2} \cdot \varepsilon$. Therefore $S q^{2+2^{1+2} \cdot \varepsilon}\left(\hat{u}_{i_{1}} \otimes \hat{u}_{i_{2}}\right)=$ $\hat{u}_{i_{1}}^{2} \otimes \hat{u}_{i_{2}}^{2}$, which contradicts the indecomposability of $u_{n-1}$. Thus $S q^{2+2^{i+2} \cdot \varepsilon} S q^{2} v_{n+1}$ does not contribute to $v_{n+1}^{2}$, and hence $S q^{4} S q^{2^{1+2} \cdot \varepsilon} v_{n+1}$ has to do contribute, since $S q^{1} v_{n+1}=0$ for dimensional reasons. Here we have

$$
S q^{2^{1+2} \varepsilon} v_{n+1} \in \operatorname{Im} \delta .
$$

So the following two cases can be considered:

$$
\begin{aligned}
& S q^{2^{+2} \cdot \varepsilon} v_{n+1}=\delta \Sigma^{4}\left(\gamma_{2^{t_{1}}} u_{q-1} \otimes \gamma_{2^{t_{2}}} u_{q-1}\right)+\text { other terms } \\
& S q^{4}\left(\gamma_{2^{i_{1}}} u_{q-1} \otimes \gamma_{2^{t_{2}}} u_{q-1}\right)=u_{n-1} \otimes u_{n-1}+\text { other terms, } \\
& S q^{2^{+2} \cdot \varepsilon} v_{n+1}=\delta \Sigma^{4}\left(\gamma_{2^{i_{1}}} u_{q-1} \otimes u_{n-1}\right)+\text { other terms } \\
& S q^{4} \gamma_{2^{i_{1}}} u_{q-1}=u_{n-1}+\text { other terms. }
\end{aligned}
$$

But the case (2) does not occur by Proposition 4.3. So the only possibility is in (1). For dimensional reasons we obtain

$$
S q^{2^{1+2} \cdot \varepsilon} v_{n+1}=\delta \Sigma^{4}\left(\gamma_{2^{\prime},} u_{q-1} \otimes \gamma_{2^{\prime},} u_{q-1}\right)+\text { other terms. }
$$

$S q^{2}\left(\gamma_{2^{\prime},} u_{q-1}\right)=u_{n-1}+$ other terms.

Comparing the dimensions we obtain $i_{1}=i$ from (b). We also have $\gamma_{2^{\prime}} u_{q-1} \in$ $\widetilde{H}^{+}\left(\Omega S^{q}\right) \subseteq \widetilde{H}^{+}(G)$, as deg $\gamma_{2^{\prime}} u_{q-1}<n-1$. Hence the element $\gamma_{2^{\prime}} u_{q-1}$ does not belong to the image of any squaring operations on $\widetilde{H}^{+}(G ; Z / 2 Z)$. 
Now we divide the arguments into the two cases, $\varepsilon=1$ and $\varepsilon=3$.

[The case $\varepsilon=3$ ] The Adem relation

$$
S q^{2^{t+2} \varepsilon}=S q^{2^{1+3}+2^{t+2}}=\sum_{t=0}^{i+2} S q^{2^{t}} a_{t}, a_{t} \in A
$$

implies that $\gamma_{2^{\prime}} u_{q-1} \otimes \gamma_{2^{\prime}} u_{q-1} \in S q^{2^{t}} a_{t}$ for some $0 \leq t \leq i+2$. On the other hand, one can deduce from $a_{t}\left(v_{n+1}\right) \in \operatorname{Im} \delta$ that $\gamma_{2^{\prime}} u_{q-1} \otimes \gamma_{2^{\prime}} u_{q-1} \in \operatorname{Im} S q^{2^{t}}$ in $H^{4}(G \wedge G ; Z / 2 Z)$ for some $t$, which contradicts the fact that $\gamma_{2} u_{q-1}$ is not in the image of any squaring operations.

[The case $\varepsilon=1$ ] If $i=1$, then $(q, n)=(3,11)$.

Suppose $i \geq 2$. By [Ad1] $S q^{2^{i+2}}$ is decomposable through secondary operations, that is, the following holds:

$$
S q^{2^{i+2}}\left(v_{n+1}\right)=\sum_{i, j} a_{i j} \Phi_{i j}\left(v_{n+1}\right), 0<\operatorname{deg} a_{i j}<2^{i+2}
$$

modulo the total indeterminacy $a_{i j k} Q^{2^{1+3}+4-l(i, j, k)}(Q(2) ; Z / 2 Z), 0<l(i, j, k)=$ $\operatorname{deg} a_{i j k}<2^{i+2}$. This leads us to a contradiction similarly to the case when $\varepsilon=3$.

This completes the proof of Theorem 4.8. Thus we obtain Theorem 4.2.

\section{§5. The Non-Existence of Types $(3,11)$ and $(7,15)$}

Proposition 5.1. $(q, n) \neq(3,11)$.

Proof. If $(q, n)=(3,11)$, then $E \simeq S^{3} \cup_{\alpha} e^{11} \cup_{\beta} e^{14}$ where $\alpha \in \pi_{10}\left(S^{3}\right) \cong$ $Z / 15$. So $E \simeq_{2}\left(S^{3} \vee S^{11}\right) \cup_{\beta} e^{14}$. Since $Q=S^{3} \vee S^{11}$ is desuspendable, the Whitehead product $[i, i]$ of the inclusion $i: Q \hookrightarrow E$ vanishes by assumption. So the map $\{i, i\}: Q \vee Q \rightarrow E$ is extendable over $Q \times Q$. We denote the extension by $\mu$ : $Q \times Q \rightarrow E$. If we put $Q(2)=C_{H(\mu)}$, the cofibre of the Hopf construction of $\mu$, then $Q(2)$ satisfies the condition of $\S 1$. It gives a contradiction, and so $(q, n) \neq$ $(3,11)$. This implies the proposition.

Proposition 5.2. $(q, n) \neq(7,15)$.

Proof. Suppose $(q, n)=(7,15)$ so that $E \simeq_{2} S^{7} \cup_{\alpha} e^{15} \cup e^{22}$. Then we have

$$
\begin{aligned}
& H^{+}(E) \cong \Lambda\left(x_{7}, x_{15}\right) \\
& K^{+}(E) \cong \Lambda\left(\xi_{7}, \xi_{15}\right) .
\end{aligned}
$$

The 15 -skeleton of $G$ is given by

$$
G^{[15]} \simeq_{2} S^{6} \cup \cup_{\left[\iota_{6}, \iota_{6}\right]} e^{12} \cup e^{14} .
$$

Now we put $Q=\Sigma\left(G^{[15]}\right) ;$ then

$$
Q \simeq_{2}\left(S^{7} \vee S^{13}\right) \underset{\bar{\alpha}}{\cup} e^{15}, \text { where } \bar{\alpha} \in \pi_{14}\left(S^{7} \vee S^{13}\right) \cong \pi_{14}\left(S^{7}\right) \oplus \pi_{14}\left(S^{13}\right) .
$$


The generators of $H^{+}(E)$ (and $K^{+}(E)$ ) are mapped monomorphically to $H^{+}(Q)$ (and $K^{+}(Q)$, resp.) by the induced homomorphism of the composite map $\ell$ : $Q \subset \Sigma G \subset P^{\infty} G \simeq E$. In fact, as was already seen, $P H^{+}(G) \cong Z_{(2)}\left\{u_{6}, u_{4}\right\}$ with $u_{i}$ transgressive, and $\ell^{+}$gives rise to the cohomology suspension. Thus we obtain

$$
\begin{aligned}
& \operatorname{Im}(\Sigma \ell)^{+} \cong Z_{(2)}\left\{v_{8}, v_{16}\right\} \subseteq H^{+}(\Sigma Q)=Z_{(2)}\left\{v_{8}, v_{14}, v_{16}\right\}, \\
& \operatorname{Im}(\Sigma \ell)^{+} \cong Z_{(2)}\left\{w_{4}, w_{8}\right\} \subseteq K^{+}(\Sigma Q)=Z_{(2)}\left\{w_{4}, w_{7}, w_{8}\right\} .
\end{aligned}
$$

Then the Adams operation $\psi^{k}$ in $K^{\dagger}(\Sigma Q)$ is given by

$$
\begin{aligned}
& \psi^{k} w_{4}=k^{4} w_{4}+a(k) w_{8} \\
& \psi^{k} w_{7}=k^{7} w_{7}+b(k) w_{8} \\
& \psi^{k} w_{8}=k^{8} w_{8}
\end{aligned}
$$

Since $Q$ is a suspended space and since $E$ is a $G W$-space, there exists an axial map

$$
\mu: Q \times Q \rightarrow E
$$

with axes $(\ell, \ell)$. We denote by $Q(2)$ the mapping cone of the Hopf construction $H(\mu)$ of the map $\mu$ so that we have a cofibre sequence

$$
\Sigma E \stackrel{j}{\hookrightarrow} Q(2) \rightarrow \Sigma Q \wedge \Sigma Q
$$

The elements $x_{7}, x_{15} \in H^{*}(E)$ are primitive with respect to $\mu$ in the sense of Thomas as $H^{11}(Q \wedge Q)=H^{15}(Q \wedge Q)=0$. Hence we have

$$
\begin{aligned}
& \bar{\mu}^{*}\left(x_{i}\right)=0 \quad \text { for } i=7,15, \\
& \bar{\mu}^{*}\left(x_{7}, x_{15}\right)=\ell^{*} x_{7} \otimes \ell^{*} x_{15}-\ell^{*} x_{15} \otimes \ell^{*} x_{7} .
\end{aligned}
$$

So the image of $j^{+}$induced by the inclusion $j: \Sigma E \rightarrow Q(2)$ is given by

$$
\operatorname{Im} j^{+}=Z_{(2)}\left\{\Sigma^{+} x_{7}, \Sigma^{+} x_{15}\right\} \text {. }
$$

Also the image of $\delta$ induced by the collapsing map $Q(2) \rightarrow \Sigma Q \wedge \Sigma Q$ is given by

$$
\operatorname{Im} \delta \cong Z_{(2)}\left\{\delta\left(v_{8} \otimes v_{8}\right), \delta\left(v_{8} \otimes v_{16}\right)=\delta\left(v_{16} \otimes v_{8}\right), \delta\left(v_{16} \otimes v_{16}\right)\right\} \oplus S_{2}
$$

where $S_{2}=Z_{(2)}\left\{\delta\left(v_{8} \otimes v_{14}\right), \delta\left(v_{14} \otimes v_{8}\right), \delta\left(v_{14} \otimes v_{14}\right), \delta\left(v_{14} \otimes v_{16}\right), \delta\left(v_{16} \otimes v_{14}\right)\right\}$.

Therefore by (5.4) we obtain the following short exact sequence:

$$
0 \rightarrow \operatorname{Im} \delta \hookrightarrow \widetilde{H}^{*}(Q(2)) \stackrel{j^{*}}{\rightarrow} Z_{(2)}\left\{\Sigma^{*} x_{7}, \Sigma^{*} x_{15}\right\} \rightarrow 0
$$

Thus, denoting by $\bar{v}_{4}$ and $\bar{v}_{8}$ the extensions over $Q(2)$ of $\Sigma^{+} x_{7}$ and $\Sigma^{+} x_{15}$, respectively, we obtain the following ring isomorphisms by virtue of [Th3]:

$$
\begin{aligned}
& H^{*}(Q(2)) \cong Z_{(2)}^{[3]}\left[\bar{v}_{4}, \bar{v}_{8}\right] \oplus S_{2}, \\
& \widetilde{H}^{*}(Q(2)) \cdot \operatorname{Im} \delta=0, S_{2} \subseteq \operatorname{Im} \delta .
\end{aligned}
$$


We remark that these results are independent of the choice of $\bar{v}_{4}$ and $\bar{v}_{8}$.

Similarly one obtains

$$
\begin{aligned}
& K^{+}(Q(2)) \cong Z_{(2)}^{[3]}\left(\bar{w}_{4}, \bar{w}_{8}\right) \oplus S_{2}^{K}, \\
& \widetilde{K}^{*}(Q(2)) \cdot S_{2}^{K}=0, \\
& \psi^{k}\left(\widetilde{K}^{*}(Q(2)) \cdot \widetilde{K}^{+}(Q(2))\right) \subseteq \widetilde{K}^{+}(Q(2)) \cdot \widetilde{K}^{+}(Q(2)), \\
& \operatorname{Im} \delta^{K} \cong Z_{(2)}\left\{\delta^{K}\left(w_{4} \otimes w_{4}\right), \delta^{K}\left(w_{4} \otimes w_{8}\right)=\delta^{K}\left(w_{8} \otimes w_{4}\right), \delta^{K}\left(w_{8} \otimes w_{8}\right)\right\} \oplus S_{2}^{K}, \\
& S_{2}^{K}=Z_{(2)}\left\{\delta^{K}\left(w_{4} \otimes w_{7}\right), \delta^{K}\left(w_{7} \otimes w_{4}\right), \delta^{K}\left(w_{7} \otimes w_{7}\right), \delta^{K}\left(w_{7} \otimes w_{5}\right), \delta^{K}\left(w_{8} \otimes w_{7}\right)\right\},
\end{aligned}
$$

where the elements $\bar{w}_{4}$ and $\bar{w}_{8}$ are the extensions over $Q(2)$ of $\Sigma^{+} \xi_{7}$ and $\Sigma^{+} \xi_{15}$, respectively.

Furthermore, by (5.3) one obtains

\section{Proposition 5.7.}

$$
\begin{aligned}
& \psi^{k} \delta^{K}\left(w_{4} \otimes w_{7}\right) \equiv k^{11} \delta^{K}\left(w_{4} \otimes w_{7}\right)+k^{4} b(k) \delta^{K}\left(w_{4} \otimes w_{8}\right) \\
& \psi^{k} \delta^{K}\left(w_{7} \otimes w_{4}\right) \equiv k^{11} \delta^{K}\left(w_{7} \otimes w_{4}\right)+k^{9} b(k) \delta^{K}\left(w_{8} \otimes w_{4}\right)
\end{aligned}
$$

modulo higher $C W$ filtration $>14$.

Now (5.5) and (5.6) imply that $K^{*}(Q(2))$ and $H^{*}(Q(2))$ are isomorphic as rings. So we define a ring isomorphism $J: H^{*}(Q(2)) \rightarrow K^{*}(Q(2))$ by the following

$$
\begin{aligned}
& J\left(\bar{v}_{i}\right)=\bar{w}_{i} \quad \text { for } i=4 \text { and } 8 \\
& J\left(\delta\left(v_{2 i} \otimes v_{2 j}\right)\right)=\delta\left(w_{i} \otimes w_{j}\right) \quad \text { for } i, j=4,7 \text { or } 8 .
\end{aligned}
$$

By virtue of these relations we introduce Hubbuck operations following [Hu] Then one obtains the following by using $(1.5)$ as in the case $(q, n)=(7,15)$ in $\$ 1$ :

$$
\begin{aligned}
& P^{8}\left(\bar{v}_{8}\right) \equiv \bar{v}_{8}^{2} \quad \bmod 2 \\
& P^{4}\left(\bar{v}_{8}\right)=\alpha \bar{v}_{4} \bar{v}_{8} \\
& P^{4}\left(\bar{v}_{4}\right) \equiv \bar{v}_{4}^{2} \quad \bmod 2 \\
& P^{4}\left(\bar{v}_{4}\right)=\lambda \bar{v}_{4}^{2}+2 \beta \bar{v}_{4} \bar{v}_{8},
\end{aligned}
$$

where $\lambda, \alpha, \beta \in Z_{(2)}$ and $\lambda \equiv 1 \bmod 2$. (Note that $J$ depends on the choice of $\bar{w}_{i}$ and hence, so do the exact values of $P^{i}$ and $R^{i}$. But these relations do not depend on the choice of $J$.)

Next, we will derive a contradiction from the relations of these Hubbuck operations. The relations

$$
H^{i}(Q(2))=0 \quad \text { for } i=10,12,14,18,20,26
$$


and Proposition 5.7 imply the following

$$
\begin{aligned}
R^{1}\left(\bar{v}_{8}\right)=P^{1}\left(\bar{v}_{8}\right)=0, & P^{1}\left(\bar{v}_{4}\right)=R^{1}\left(\bar{v}_{4}\right)=0, \\
R^{2}\left(\bar{v}_{8}\right)=P^{2}\left(\bar{v}_{8}\right)=0, P^{2}\left(\bar{v}_{4}\right) & =R^{2}\left(\bar{v}_{4}\right)=0, \\
P^{3}\left(\bar{v}_{4}\right) & =R^{3}\left(\bar{v}_{4}\right)=0, \\
P^{5}\left(\bar{v}_{8}\right)=0, & P^{5}\left(\bar{v}_{4}\right)=0, \\
P^{6}\left(\bar{v}_{4}\right) & =0 .
\end{aligned}
$$

Further, by (1.4) together with $v_{2}\left(3^{3}-1\right)=1$ (by ignoring the odd multiple) one has

$2 P^{3}\left(\bar{v}_{8}\right)+2 R^{1} P^{2}\left(\bar{v}_{8}\right)+2^{2} R^{2} P^{1}\left(\bar{v}_{8}\right)+2^{3} R^{3}\left(\bar{v}_{8}\right) \equiv 2^{2} P^{2} R^{1}\left(\bar{v}_{8}\right)+2^{4} P^{1} R^{2}\left(\bar{v}_{8}\right) \bmod 2^{6}$ and hence by (5.10) one obtains the following

$$
2 P^{3}\left(\bar{v}_{8}\right)+2^{3} R^{3}\left(\bar{v}_{8}\right) \equiv 0 \quad \bmod 2^{6} .
$$

In particular

$$
P^{3}\left(\bar{v}_{8}\right) \equiv 0 \quad \bmod 2^{2}
$$

Also, (1.4) implies

$$
\left(2^{4} P^{4}+\sum_{i=1}^{4} 2^{i} R^{i} P^{4-i}\right)\left(\bar{v}_{4}\right) \equiv 2^{2} P^{3} R^{1}\left(\bar{v}_{4}\right)+2^{4} P^{2} R^{2}\left(\bar{v}_{4}\right) \quad \bmod 2^{6}
$$

and hence one obtains the following

$$
P^{4}\left(\bar{v}_{4}\right)+R^{4}\left(\bar{v}_{4}\right) \equiv 0 \quad \bmod 2^{2} .
$$

Moreover one obtains

Proposition 5.13.

$$
P^{6}\left(\bar{v}_{8}\right) \equiv 2^{3} R^{6}\left(\bar{v}_{8}\right) \quad \bmod 2^{4}
$$

Proof. The equation (1.4) implies

$2^{3} P^{6}\left(\bar{v}_{8}\right)+\sum_{i=1}^{6} 2^{i} R^{i} P^{6-i}\left(\bar{v}_{8}\right) \equiv 2^{2} P^{5} R^{1}\left(\bar{v}_{8}\right)+2^{4} P^{4} R^{2}\left(\bar{v}_{8}\right)+2^{6} P^{3} R^{3}\left(\bar{v}_{8}\right) \bmod 2^{7}$

Recall that $P^{4}\left(\bar{v}_{8}\right) \in Z_{(2)}\left\{\bar{v}_{4} \bar{v}_{8}\right\}$, where we have

$$
R^{2}\left(\bar{v}_{4} \bar{v}_{8}\right)=R^{2}\left(\bar{v}_{4}\right) \bar{v}_{8}+R^{1}\left(\bar{v}_{4}\right) R^{1}\left(\bar{v}_{8}\right)+\bar{v}_{4} R^{2}\left(\bar{v}_{8}\right)=0
$$

and hence $R^{2} P^{4}\left(\bar{v}_{8}\right)=0$. So by (5.10) and (5.11) the congruence equation above reduces to

$$
2^{3} P^{6}\left(\bar{v}_{8}\right)+2^{5} R^{3} R^{3}\left(\bar{v}_{8}\right)+2^{6} R^{6}\left(\bar{v}_{8}\right) \equiv 2^{6} P^{3} R^{3}\left(\bar{v}_{8}\right) \quad \bmod 2^{7}
$$

where $R^{3}\left(\bar{v}_{8}\right) \in Z_{(2)}\left\{\delta\left(v_{8} \otimes v_{14}\right), \quad \delta\left(v_{14} \otimes v_{8}\right)\right\}$. Hence by (5.10) we have $R^{3} R^{3}\left(\bar{v}_{8}\right)=P^{3} R^{3}\left(\bar{v}_{8}\right)=0$. Thus the congruence equation above reduces to 


$$
P^{6}\left(\bar{v}_{8}\right)+2^{3} R^{6}\left(\bar{v}_{8}\right) \equiv 0 \quad \bmod 2^{4} .
$$

This implies the proposition.

\section{Proposition 5.14.}

$$
2 P^{8}\left(\bar{v}_{8}\right) \equiv R^{4} P^{4}\left(\bar{v}_{8}\right) \quad \bmod 4
$$

Proof. The equation (1.4) implies

$$
2 P^{7}\left(\bar{v}_{8}\right)+\sum_{i=1}^{5} 2^{i} R^{i} P^{7-i}\left(\bar{v}_{8}\right) \equiv 2^{2} P^{6} R^{1}\left(\bar{v}_{8}\right)+2^{4} P^{5} R^{2}\left(\bar{v}_{8}\right) \bmod 2^{6} .
$$

So by using (5.10), (5.11') and Proposition 5.13 one obtains

$$
2 P^{7}\left(\bar{v}_{8}\right)+2^{4} R^{1} R^{6}\left(\bar{v}_{8}\right)+2^{3} R^{3} P^{4}\left(\bar{v}_{8}\right) \equiv 0 \bmod 2^{6},
$$

where $P^{4}\left(\bar{v}_{8}\right) \in Z_{(2)}\left\{\bar{v}_{4} \bar{v}_{8}=\delta\left(v_{8} \otimes v_{16}\right)\right\} \subseteq \widetilde{H}^{*}(Q(2)) \cdot \widetilde{H}^{*}(Q(2))$, and hence

$$
R^{3} P^{4}\left(\bar{v}_{8}\right) \in Z_{(2)}\left\{R^{3}\left(\bar{v}_{4} \bar{v}_{8}\right)\right\} .
$$

By (5.10) and the Cartan formula we have

$$
R^{3}\left(\bar{v}_{4} \bar{v}_{8}\right)=\bar{v}_{4} R^{3}\left(\bar{v}_{8}\right)
$$

with $R^{3}\left(\bar{v}_{8}\right) \in S_{2}$. So by (5.5) we have $R^{3} P^{4}\left(\bar{v}_{8}\right)=0$. Therefore we obtain

$$
2 P^{7}\left(\bar{v}_{8}\right)+2^{4} R^{1} R^{6}\left(\bar{v}_{8}\right) \equiv 0 \bmod 2^{6}
$$

Also the equation (1.4) implies

(5.16) $2^{5} P^{8}\left(\bar{v}_{8}\right)+\sum_{i=1}^{5} 2^{i} R^{i} P^{8-i}\left(\bar{v}_{8}\right) \equiv 2^{2} P^{7} R^{1}\left(\bar{v}_{8}\right)+2^{4} P^{6} R^{2}\left(\bar{v}_{8}\right) \bmod 2^{6}$

Then by (5.10), (5.11'), Proposition 5.13 and (5.15), one obtains

$$
2^{5} P^{8}\left(\bar{v}_{8}\right)+2^{4} R^{1} R^{1} R^{6}\left(\bar{v}_{8}\right)+2^{5} R^{2} R^{6}\left(\bar{v}_{8}\right)+2^{4} R^{4} P^{4}\left(\bar{v}_{8}\right) \equiv 0 \bmod 2^{6} .
$$

From (1.4) it follows that

$$
2 P^{1}+2 R^{1} \equiv 2^{2} R^{1}, \quad \bmod 2^{3}
$$

and hence $P^{1} \equiv \pm R^{1} \bmod 2^{2}$. Also from (1.4), one has

$$
2^{3} P^{2}+2 R^{1} P^{1}+2^{2} R^{2} \equiv 2^{2} P^{1} R^{1}+2^{4} R^{2} \bmod 2^{3} \text {. }
$$

Then it follows that

$$
R^{1} R^{1}=2 R^{2} \bmod 2^{2}
$$

Hence

$$
R^{1} R^{1} R^{6}\left(\bar{v}_{8}\right)+2 R^{2} R^{6}\left(\bar{v}_{8}\right) \equiv 0 \bmod 2^{2}
$$


Substituting this into (5.17) one obtains

$$
2^{5} P^{8}\left(\bar{v}_{8}\right)+2^{4} R^{4} P^{4}\left(\bar{v}_{8}\right) \equiv 0 \bmod 2^{6} .
$$

By dividing by $2^{4}$, we obtain Proposition 5.14.

Proposition 5.18. Let $\beta$ be as in (5.9). If $\beta \neq \equiv \bmod 2, R^{4} P^{4}\left(\bar{v}_{4}\right) \equiv 0 \bmod 4$.

Proof. The equation (1.4) implies

$$
2^{5} P^{8}\left(\bar{v}_{4}\right)+\sum_{i=1}^{5} 2^{i} R^{i} P^{8-i}\left(\bar{v}_{4}\right) \equiv 2^{2} P^{7} R^{1}\left(\bar{v}_{4}\right)+2^{4} P^{6} R^{2}\left(\bar{v}_{4}\right) \quad \bmod 2^{6} .
$$

So by (1.5) and (5.10) one obtains

$$
2^{2} R^{1} P^{7}\left(\bar{v}_{4}\right)+2^{4} R^{4} P^{4}\left(\bar{v}_{4}\right) \equiv 0 \quad \bmod 2^{6} .
$$

Furthermore (1.4) implies

$$
2 P^{7}\left(\bar{v}_{4}\right)+\sum_{i=1}^{5} 2^{i} R^{i} P^{7-i}\left(\bar{v}_{4}\right) \equiv 2^{2} P^{6} R^{1}\left(\bar{v}_{4}\right)+2^{4} P^{5} R^{2}\left(\bar{v}_{4}\right) \quad \bmod 2^{6} .
$$

So by (5.10) one obtains

$$
2 P^{7}\left(\bar{v}_{4}\right)+2^{3} R^{3} P^{4}\left(\bar{v}_{4}\right) \equiv 0 \bmod 2^{6} .
$$

Recall from (5.9) that

$$
P^{4}\left(\bar{v}_{4}\right)=\lambda \bar{v}_{4}^{2}+2 \beta \bar{v}_{8}
$$

So by (5.10) one has

$$
R^{3} P^{4}\left(\bar{v}_{4}\right)=2 \beta R^{3}\left(\bar{v}_{8}\right)
$$

Suppose $\beta \neq \equiv 0 \bmod 2$. By (1.5), one has $P^{7}\left(\bar{v}_{4}\right) \equiv 0 \bmod 2^{4}$ and hence by (5.20) one obtains

$$
2^{3} R^{3} P^{4}\left(\bar{v}_{4}\right) \equiv 0 \quad \bmod 2^{5},
$$

so $2^{4} \beta R^{3}\left(\bar{v}_{8}\right) \equiv 0 \bmod 2^{5}$. Thus

$$
R^{3}\left(\bar{v}_{8}\right) \equiv 0 \bmod 2 .
$$

Then it follows from (5.11) that

$$
P^{3}\left(\bar{v}_{8}\right) \equiv 2^{2} R^{3}\left(\bar{v}_{8}\right) \equiv 0 \bmod 2^{3} .
$$

So by rechoosing the ring isomorphism $J$ appropriately (or more precisely, rechoosing the extension $\bar{w}_{8}=J\left(\bar{v}_{8}\right)$ appropriately) one obtains the following lemma (due to $[\mathrm{Hu}]$ ).

Lemma 5.23. One can choose a ring isomorphism $J$ which satisfies $P_{J}^{3}\left(\bar{v}_{8}\right)=0$, if $\beta \neq 0$ mod 2 . 
Proof. If $P^{3}\left(\bar{v}_{8}\right) \neq 0$, we can choose $\bar{v}_{11} \in H^{22}(Q(2))$ so that $P^{3}\left(\bar{v}_{8}\right)=2^{3} \bar{v}_{11}$. The element $\bar{w}_{8}^{\prime}=\bar{w}_{8}+v \bar{w}_{11}$ with $v=\frac{1}{1-2^{3}}$, where $\bar{w}_{11}=J\left(\bar{v}_{11}\right)$, is an extension of $\Sigma^{+} \xi_{15}$. Then from $J$, we define a new ring isomorphism $J^{\prime}: H^{*}(Q(2)) \rightarrow K^{*}(Q(2))$ by setting

$$
\begin{aligned}
& J^{\prime}\left(\bar{v}_{8}\right)=\bar{w}_{8}^{\prime}, J^{\prime}\left(\bar{v}_{4}\right)=\bar{w}_{4} \\
& J^{\prime}\left(\delta\left(v_{2 i} \otimes v_{2 j}\right)\right)=\delta^{K}\left(w_{i} \otimes w_{j}\right) .
\end{aligned}
$$

Then one obtains the following formula modulo higher filtration $>11$ :

$$
\begin{aligned}
\psi^{2}\left(J\left(\bar{v}_{8}\right)\right) & \equiv 2^{8} J\left(\bar{v}_{8}\right)+2^{8} J\left(\bar{v}_{11}\right) \quad \bmod (\text { higher filtration }>11) \\
\psi^{2}\left(J\left(\bar{v}_{11}\right)\right) & \equiv 2^{11} J\left(\bar{v}_{11}\right) \quad \bmod (\text { higher filtration }>11) \\
\psi^{2}\left(J^{\prime}\left(\bar{v}_{8}\right)\right) & =\psi^{2}\left(J\left(\bar{v}_{8}\right)+v J\left(\bar{v}_{11}\right)\right) \\
& =\psi^{2}\left(J\left(\bar{v}_{8}\right)\right)+v \psi^{2}\left(J\left(\bar{v}_{11}\right)\right) \\
& \equiv 2^{8} J\left(\bar{v}_{8}\right)+2^{8} J\left(\bar{v}_{11}\right)+2^{11} v J\left(\bar{v}_{11}\right) \quad \bmod (\text { higher filtration }>11) \\
& \equiv 2^{8}\left(J\left(\bar{v}_{8}\right)+\left(2^{3} v+1\right) J\left(\bar{v}_{11}\right)\right) \quad \bmod (\text { higher filtration }>11) \\
& =2^{8} J^{\prime}\left(\bar{v}_{8}\right) .
\end{aligned}
$$

Thus $P_{J^{\prime}}^{3}\left(\bar{v}_{8}\right)=0$. (Note that the operation $P_{J^{\prime}}^{3}$ with respect to $J^{\prime}$ is different from $P^{3}=P_{J}^{3}$ with respect to $\left.J\right)$. The operations $P_{J}^{i}$, and $R_{J}^{i}$, satisfy all the formulae given above for the ones with respect to general ' $J$ '. So, we may assume that our ring isomorphism $J$ satisfies $P_{J}^{3}=0$. This implies the lemma.

Hence from (5.11), (5.21) and (5.20) it follows that

$$
\begin{aligned}
& R^{3}\left(\bar{v}_{8}\right) \equiv 0 \bmod 2^{3}, \\
& R^{3} P^{4}\left(\bar{v}_{4}\right) \equiv 0 \quad \bmod 2^{4} \text {, } \\
& 2 P^{7}\left(\bar{v}_{4}\right) \equiv 0 \bmod 2^{6} \text {. }
\end{aligned}
$$

Substituting them into (5.19) one obtains

$$
2^{4} R^{4} P^{4}\left(\bar{v}_{4}\right) \equiv 0 \quad \bmod 2^{6} .
$$

That is, if $\beta \equiv 0 \bmod 2$, then $R^{4} P^{4}\left(\bar{v}_{4}\right) \equiv 0 \bmod 4$. This completes the proof of Proposition 5.18.

Now these two propositions, Propositions 5.14 and 5.18, will give us a contradiction in the following manner:

By Proposition 5.14, we have the following equation

(5.24) $0 \not \equiv 2 \bar{v}_{8}^{2} \equiv R^{4} P^{4}\left(\bar{v}_{8}\right) \equiv R^{4}\left(\alpha \bar{v}_{4} \bar{v}_{8}\right) \equiv \alpha R^{4}\left(\bar{v}_{4}\right) \bar{v}_{8}+\alpha \bar{v}_{4} R^{4}\left(\bar{v}_{8}\right) \quad \bmod 4$

by (5.10) and the Cartan formula, where $R^{4}\left(\bar{v}_{8}\right) \in \operatorname{Im} \delta$ and hence $\bar{v}_{4} R^{4}\left(\bar{v}_{8}\right)=0$ by (5.5). Furthermore, using (5.10) together with (1.4), one obtains the following relation:

$$
2^{4} P^{4}\left(\bar{v}_{4}\right)+2^{4} R^{4}\left(\bar{v}_{4}\right) \equiv 0 \quad \bmod 2^{6}
$$


which implies

$$
R^{4}\left(\bar{v}_{4}\right) \equiv-P^{4}\left(\bar{v}_{4}\right) \equiv-\lambda \bar{v}_{4}^{2}-2 \beta \bar{v}_{8} \quad \bmod 4 .
$$

Hence from (5.24) it follows that

$$
0 \neq 2 \bar{v}_{8}^{2} \equiv-2 \alpha \beta \bar{v}_{8}^{2} \bmod 4 .
$$

Then it follows that

$$
\alpha \beta \equiv 1 \bmod 2 ; \text { in particular, } \beta \equiv 1 \bmod 2 .
$$

Since $\beta \neq \equiv 0 \bmod 2$, Proposition 5.18 implies

$$
0 \equiv R^{4} P^{4}\left(\bar{v}_{4}\right) \equiv R^{4}\left(\lambda \bar{v}_{4}^{2}+2 \beta \bar{v}_{8}\right) \equiv 2 \lambda \bar{v}_{4} R^{4}\left(\bar{v}_{4}\right)+2 \beta R^{4}\left(\bar{v}_{8}\right) \quad \bmod 4
$$

by (5.10) and the Cartan formula. Here, by (5.25), we have

$$
2 \lambda \bar{v}_{4} R^{4}\left(\bar{v}_{4}\right) \equiv 0 \quad \bmod 4
$$

Also by (1.4) using (5.10) and Lemma 5.23 we have

$$
2^{4} P^{4}\left(\bar{v}_{8}\right)+2^{4} R^{4}\left(\bar{v}_{8}\right) \equiv 0 \bmod 2^{6}
$$

and hence

$$
R^{4}\left(\bar{v}_{8}\right) \equiv-P^{4}\left(\bar{v}_{8}\right)=-\alpha \bar{v}_{4} \bar{v}_{8} \quad \bmod 4
$$

Substituting them into (5.27) we obtain

$$
0 \equiv R^{4} P^{4}\left(\bar{v}_{4}\right) \equiv-2 \alpha \beta \bar{v}_{4} \bar{v}_{8} \quad \bmod 4,
$$

which contradicts (5.26).

Thus we have shown that there exists no Poincaré complex with GW-space structure whose cohomology ring is an exterior algebra of type $(7,15)$. This completes the proof of Proposition 5.2.

\section{§6. Proof of the Main Theorem}

In this section, we always assume that $E$ is a complex of type $(q, n, m)$. Let us assume that $E$ has a cell structure $S^{q} \cup_{\alpha} e^{n} \cup_{\beta} e^{m}$ with $\alpha \in \pi_{n-1}\left(S^{q}\right), \beta \in$ $\pi_{m-1}\left(S^{q} \cup_{\alpha} e^{n}\right)$. At first, we look at cohomological structure of $E$.

Proposition 6.1. Let $E$ be a $G W$-space at $\Pi$ where $\Pi$ is a set of primes. If $\alpha=0$ at $\Pi$, then $E \simeq S^{t}$ with $t$ odd or $S^{q} \times S^{n}$ with $q$ and $n$ odd. If further $2 \in \Pi$, we have $t \in\{1,3,7\}$ and $\{q, n\} \subset\{1,3,7\}$.

Proof. We will prove here the integral case. The localised version can be obtained by a quite similar manner and is left to the reader.

Since $\alpha=0, E \simeq\left(S^{q} \vee S^{n}\right) \cup_{\beta} e^{m}$. We denote by $i_{1}: S^{q} \hookrightarrow E$ and $i_{2}: S^{n} \longrightarrow E$ 
the canonical inclusions. Since a sphere is desuspendable, by $(0.3)$, there is an axial map $v: S^{q} \times S^{n} \rightarrow E$ with axes $\left(i_{1}, i_{2}\right)$ by the assumption. We remark here that the attaching map of the top cell of $S^{q} \times S^{n}$ is given by the Whitehead product $\left[i_{1}, i_{2}\right]$.

Since $E$ has cells only in dimensions $0, q, n$ and $m$, there are three possibilities on its cohomology: $H^{+}(E ; Z) \cong Z \oplus Z \oplus Z, Z \oplus Z / r Z$ (for some $r>1$ ) or $Z$. In the last case, $m$ has to be $q+1\left(n+1\right.$, resp.). Then $H^{+}(E ; Z)$ is isomorphic to $H^{+}\left(S^{n} ; Z\right)\left(H^{+}\left(S^{q} ; Z\right)\right.$, resp. $)$ which is given by $i_{2}^{+}\left(i_{1}^{+}\right.$, resp. $)$. Since $E$ is simple, $E$ has the homotopy type of $S^{n}\left(S^{q}\right.$, resp.).

Let us recall that a sphere is a GW-space (at 2) if and only if it is $S^{1}, S^{3}$ or $S^{7}$ by [Ad1]. Thus $n(q$, resp. $)=1,3$ or 7 .

In the other cases, $i_{1}$ and $i_{2}$ induce non-trivial homomorphisms of cohomologies for some coefficient ring $Z / p Z, p$ a prime. Then $v^{+}$is a surjection, since the generators in $H^{+}\left(S^{q} \times S^{n} ; Z / p Z\right)$ are in its image. Thus we obtain that $H^{q+n}(E ; Z / p Z) \neq 0$, and hence $m=q+n>n$. If $H^{+}(E ; Z) \cong Z \oplus Z / r Z$, then the action of some higher order Bockstein operation is not trivial on $H^{+}(E$; $Z / p Z)$ for some prime $p$, but is trivial on $H^{+}\left(S^{q} \times S^{n} ; Z / p Z\right)$ for any $p$. It is a contradiction and we have $H^{+}(E ; Z)=Z \oplus Z \oplus Z$. Hence $v^{+}$is an isomorphism. Since $E$ is simple, $E$ has the homotopy type of $S^{q} \times S^{n}$, which is a Poincaré complex and a GW-space. Also the mod 2 Steenrod algebra acts trivially on $H^{+}(E ; Z / 2 Z)$. Then by the argument given in the proof of Corollary 1.9 , one has $\{q, n\} \subset\{1,3,7\}$.

This implies the proposition.

Proposition 6.2. Let $n>q>1$ and $p$ a prime. If $H^{j}(E ; Z / p Z)$ are non-zero for $j=q$ and $n$, then $E$ is a Poincaré complex of type $(q, n, q+n)$.

Proof. Let $Q$ be the following suspended subspace of $\Sigma \Omega E$ and $l: Q \rightarrow E$ be the composite map $Q \subset \Sigma G \subset P^{\infty} G \simeq E$ :

$$
\begin{gathered}
Q=Q^{[n-1]} \cup e^{n} \subseteq \Sigma(\Omega E)^{[n-1]}, \\
Q^{[n-1]} \simeq \bigvee_{\iota=1}^{\left[\frac{n-2}{q-1}\right]} S^{i(q-1)+1} \subseteq \Sigma(\Omega E)^{[n-2]} .
\end{gathered}
$$

Then by the Serre spectral sequence for the fibration $G \rightarrow P E \rightarrow E$, one obtains, similarly to the proof of Proposition $3.3(2)$, that $\sigma^{+} x_{q}$ and $\sigma^{+} x_{n}$ are non-zero primitive generators in dimensions $q-1$ and $n-1$ in $H^{+}(G ; Z / p Z)$. Since $l$ induces the cohomology suspension, $l^{+}$is an isomorphism in dimensions $q$ and $n$.

Since $Q$ and $S^{q}$ are suspended spaces, there exists an axial map $\mu: Q \times S^{q} \rightarrow E$ with axes $\left(l,\left.l\right|_{S^{4}}\right)$. Then $\mu$ induces a homomorphism $\mu^{+}: H^{+}(E ; Z / p Z) \rightarrow$ $H^{*}\left(Q \times S^{q} ; Z / p Z\right) \cong H^{+}(Q ; Z / p Z) \otimes H^{+}\left(S^{q} ; Z / p Z\right)$. We have 


$$
\begin{aligned}
& \mu^{+}\left(x_{q}\right)=l^{+}\left(x_{q}\right) \otimes 1+1 \otimes l^{+}\left(x_{q}\right), \\
& \mu^{+}\left(x_{n}\right)=l^{+}\left(x_{n}\right) \otimes 1+y_{n-q} \otimes l^{+}\left(x_{q}\right)
\end{aligned}
$$

for some $y_{n-q} \in H^{n-q}(Q ; Z / p Z)$.

Then we obtain that $\mu^{+}\left(x_{n} x_{q}\right)=l^{+}\left(x_{n}\right) \otimes l^{+}\left(x_{q}\right)+l^{+}\left(x_{q}\right) y_{n-q} \otimes l^{+}\left(x_{q}\right)=$ $l^{+}\left(x_{n}\right) \otimes l^{+}\left(x_{q}\right) \neq 0$, since $Q$ is suspended. Thus $x_{n} x_{q} \neq 0$ and hence $H^{q+n}(E$; $Z / p Z) \neq 0$. This implies that $m=q+n>n+1$ and we obtain

$$
E \simeq S^{q} \cup_{\alpha} e^{n} \cup_{\beta} e^{q+n} .
$$

Moreover we obtain that

$$
\begin{aligned}
& H^{j}(E ; Z / r Z) \cong Z / r Z\left\{x_{j}\right\} \quad \text { for } j=q, q+1 \text { and } 2 q+1, \\
& H^{q}(E ; Z) \cong 0, H^{n}(E ; Z) \cong Z / r Z, H^{q+n}(E ; Z) \cong Z,
\end{aligned}
$$

if $n=q+1$ and $\alpha=r \iota_{q}$ with $r \neq \pm 1$ nor 0 , and that

$$
H^{j}(E ; Z) \cong Z\left\{x_{j}\right\} \quad \text { for } j=q, n \text { and } q+n,
$$

otherwise.

Let us turn our attention to the top cells of $Q \times S^{q}$ and $E$. We have shown

(6.3). In the case $\alpha=r \iota_{q}$ with $r \neq \pm 1$ nor $0, \mu^{*}$ is an isomorphism of mod $r$ cohomology in dimension $q+n$. In other cases, by comparing integral cohomologies by $\mu^{+}$similarly to the above, we obtain that $\mu^{+}$is an isomorphism of integral cohomology in dimension $q+n$.

This implies that $E$ has an (orientation) class in dimension $q+n$ which induces a Poincaré duality. Thus $E$ is a Poincaré complex of type $(q, n, q+n)$.

This implies the proposition.

We can now state the key lemma to our main theorem, which is first known to H. Kachi for simply connected case.

Lemma 6.4. If $E$ is a $G W$-space (at 2), then it has the homotopy type (at 2) of either a sphere of dimension 1, 3 or 7, or a 3-cell Poincaré complex of type ( $q$, $n, q+n)$ of exactly $q+n$ dimension.

Proof. We show here the proof of the integral case, since the localised case at 2 is obtained by just localising the argument of the integral case.

Let $q=1$. When $n=1$ or $n>2$, it clearly holds that $\alpha$ is trivial.

If $\alpha$ is trivial, it follows from Proposition 6.1 that $E$ has a homotopy type of either $S^{1}$ or a product of $S^{1}$ and $S^{n}$ with $n \in\{1,3,7\}$ which is a Poincaré complex of type $(1, n, 1+n)$. 
If $n=2$ and $\alpha= \pm \iota_{1}$, then $E \simeq S^{m}$ with $m \geq 2$, which must be a GW-space, and hence $m \in\{3,7\}$.

If $n=2, \alpha=p \iota_{1}$ and $p \neq \pm 1$ nor 0 , then $\pi_{1}(E) \cong Z / p Z$. Then $S^{1} \cup_{\alpha} e^{2} \subset E$ is nothing but $L^{2}(p)$, the 2-skeleton of the standard lens space $L^{3}(p)$, and is not simple (nor nilpotent): Let $\pi=\left\langle\tau \mid \tau^{p}=1\right\rangle \cong \pi_{1}\left(L^{2}(p)\right)=\pi_{1}(E) \cong Z / p Z$. The universal covering space of $L^{2}(p)$ has the homotopy type of a wedge sum of $(p-1)$ copies of 2 -spheres:

$$
\begin{aligned}
& \pi_{1}\left(L^{2}(p)\right) \cong Z / p Z \tau, \\
& \pi_{2}\left(L^{2}(p)\right) \cong \sum_{i=1}^{p-1} Z \alpha_{i} .
\end{aligned}
$$

The action of a generator $\tau \in \pi$ on $\pi_{2}\left(L^{2}(p)\right)$ is given as follows:

$$
\pi_{2}\left(L^{2}(p)\right) \cong \sum_{i=1}^{p-1} Z \alpha_{i} \cong Z \pi /\left(1+\tau+\ldots+\tau^{p-1}\right), \tau \alpha_{i}=\alpha_{i+1}, \text { for } i \leq p-1,
$$

where $\alpha_{p}=-\sum_{j=1}^{p-1} \alpha_{j}$.

If $m=2$, a similar argument given in the above yields that $E$ is not simple. It contradicts the assumption that $E$ is a GW-space. Thus $m>2$ and we have the following exact sequence:

$$
\pi_{3}\left(E, L^{2}(p)\right) \rightarrow \pi_{2}\left(L^{2}(p)\right) \rightarrow \pi_{2}(E) \rightarrow 0 .
$$

Since $E$ is simple, $\tau$ acts trivially on $\pi_{2}(E)$. Then it follows that $\pi_{2}(E)$ is a quotient group of $\pi_{2}\left(L^{2}(p)\right) / \pi \cong Z / p Z$. Thus $\pi_{3}\left(E, L^{2}(p)\right) \neq 0$, and hence $m=3$.

Then it follows that $H^{+}(E ; Z) \cong H^{+}\left(L^{3}(p) ; Z\right)$ as modules.

Let us consider the Serre spectral sequence associated with the fibration $\widetilde{E} \rightarrow E \rightarrow B(Z / . p Z)$, where $\widetilde{E} \rightarrow E$ denotes the universal covering. Since $E$ is simple, so is the fibration and $H_{2}(E ; Z) \cong \pi_{2}(E)$ is finite. A routine computation on the $E_{2}$ term of the Serre spectral sequence shows that the only non-trivial differential is $d_{4}$, which yields that $H^{*}(\widetilde{E} ; Z) \cong H^{+}\left(S^{3} ; Z\right)$ as algebrae.

Since $\widetilde{E}$ is simply connected, $\widetilde{E}$ is a homotopy 3 -sphere. Hence the boundary homomorphism $\partial: \pi_{3}\left(E, L^{2}(p)\right) \rightarrow \pi_{2}\left(L^{2}(p)\right)$ is surjective by (6.7) and preserves the actions of $\tau \in \pi$ on $\pi_{3}\left(E, L^{2}(p)\right)$ :

$$
\pi_{3}\left(E, L^{2}(p)\right) \cong \sum_{i=1}^{p} Z \beta_{i} \cong Z \pi, \tau \beta_{i}=\beta_{i+1}, \quad \text { for } i \leq p,
$$

where $\beta_{p+1}=\beta_{1}$ which corresponds to the 3-cell of $E$. Thus $\beta=\partial \beta_{1}$ is a unit in $\pi_{2}\left(L^{2}(p)\right)$. We remark that the direct summand generated by $\sum_{j=1}^{p} \beta_{j}$ in $\pi_{3}(E$, $\left.L^{2}(p)\right)$ is the kernel of $\partial$, since there is no element in $\pi_{2}\left(L^{2}(p)\right)$ other than 0 to be stable under the action of $\tau$ by $(6.6)$.

Again by the Serre spectral sequence $\bmod p$ associated with the fibration $\widetilde{E} \rightarrow E \rightarrow B(Z / p Z)$, it follows that $H^{+}(E ; Z / p Z) \cong H^{+}\left(L^{3}(p) ; Z / p Z\right)$ as algebrae. This implies that $E$ is a Poincaré complex of type $(1,2,3)$. 
Conversely, let $\ell$ be a unit in $\pi_{2}\left(L^{2}(p)\right)$, which is also a unit in $\pi_{2}\left(L^{2}(p)\right) / \pi \cong$ $Z / p Z$. Let $L^{3}(p, \ell)$ be the space given by attaching a 3-cell by $\ell$ on $L^{2}(p)$. Then its cohomology ring mod $p$ is isomorphic to $H^{+}\left(L^{3}(p) ; Z / p Z\right)$, since the universal covering space is a homotopy 3 -sphere. Thus it is a Poincaré complex of type (1, 2,3).

Let $q$ be odd $>1$. Unless $n=q+1, \alpha$ has a finite order.

If $\alpha$ is trivial, it follows from Proposition 6.1 that $E$ has the homotopy type of a $q$-sphere $(q \in\{3,7\}$, since $q$ is odd $>1)$ or $S^{q} \times S^{n}(\{q, n\} \subset\{3,7\})$.

If $n=q$, then $\alpha$ is trivial and $E$ has the homotopy type of either $S^{q}$ or $S^{q} \times S^{q}, q \in\{3,7\}$.

If $n=q+1$ and $\alpha= \pm \iota_{q}$, then $E$ has the homotopy type of $m$-sphere with $q+1 \leq m=7$.

If $n=q+1$ and $\alpha=p \iota_{q}$ with $p \neq \pm 1$ nor 0 , then $H^{j}(E ; Z / p Z)$ are non-zero when $j=q$ and $n$. Then by Proposition 6.2, we obtain that $E$ is a Poincaré complex of type $(q, n, q+n)$.

If $n>q+1$ and $\alpha \neq 0$, then $\alpha$ has a finite order $>1$. Hence by Proposition 6.1, we obtain that $E$ has the rational homotopy type of a sphere $S^{q}$ or the product of spheres $S^{q} \times S^{n}$. In the former case, we have $m=n+1$ and the homomorphism $\pi_{n}\left(S^{q} \cup e^{n}\right) \rightarrow \pi_{n}\left(S^{n}\right)$ induced from the collapsion to the $n$-cell sends $\beta$ to an integer $\equiv 0 \bmod p$ where $p$ is the order of $\alpha$. Thus $H^{j}(E ; Z / p Z)$ is non-zero when $j=n$ (and $j=q$ ). Then by Proposition 6.2, we obtain that $E$ is a Poincaré complex of type $(q, n, q+n)$.

Let $q$ be even $>0$. Unless $n=q+1$ or $2 q, \alpha$ has a finite order.

If $\alpha$ is trivial, it follows from Proposition 6.1 that $E$ has the homotopy type of a $q+1$-sphere and $n=m=q+1 \in\{3,7\}$, since $q$. is even.

If $n=q$, then $\alpha$ is trivial and hence $n=q+1$. It is a contradiction.

If $n=q+1$ and $\alpha= \pm \iota_{1}$, then $E$ has the homotopy type of an $m$-sphere with $q+1 \leq m \in\{3,7\}$.

If $n=q+1$ and $\alpha=p \iota_{1}, p \neq \pm 1$ nor 0 , then by Proposition 6.2 we obtain that $E$ is a Poincaré complex of type $(q, n, q+n)$.

If $q+1<n \neq 2 q$ or $\alpha$ has a finite order, then $E$ has the rational homotopy type of either an odd sphere or a product of two odd spheres. It is impossible.

If $n=2 q$ and $\alpha$ has an infinite order, then $E$ has the rational homotopy type of $J_{2}\left(S^{q}\right) \cup_{\beta} e^{m}$, where we denote by $J_{t}(X)$ the James' ( $t$-fold) reduced product space of $X$.

Let us recall that $\pi_{t}\left(J_{2}\left(S^{q}\right)\right) \otimes Q=0$, unless $t=q$ or $3 q-1$. Thus $\beta$ has a finite order, unless $t=q$ or $3 q-1$.

Let us assume that $\beta$ has a finite order and hence $E$ has the rational homotopy type of $J_{2}\left(S^{q}\right) \vee S^{m}$. We can choose maps $i_{1}: S^{q} \longrightarrow E$ and $i_{2}: S^{m} \longrightarrow E$ which are rationally the canonical inclusions. Since a sphere is desuspendable, by (0.3), there is rationally an axial map $\mu: S^{q} \times S^{m} \rightarrow E$ with axes $\left(i_{1}, i_{2}\right)$ by the assumption. 
By the definition, we have that $i_{1}$ and $i_{2}$ induce non-trivial homomorphisms of rational cohomologies. Hence $\mu$ induces a surjection, since the generators in $H^{4}\left(S^{q} \times S^{n} ; Q\right)$ are in its image. This implies that the product of generators in dimensions $q$ and $m$ is non-zero in $H^{q+m}(E ; Q)$, but it is impossible. Thus $\beta$ is rationally non-trivial. Since $m \geq 2 q$, it follows that $m=3 q$ and $\beta$ is rationally non-trivial, $E$ has the rational homotopy type of $J_{3}\left(S^{q}\right)$. Hence by Proposition 6.2 , we obtain that $E$ is a Poincaré complex of type $(q, 2 q, 3 q)$.

This completes the proof of the lemma.

Using the above, we show the proof of the main theorem.

We may assume by Lemma 6.4 that $E$ is actually a 3-cell Poincaré complex of type $(q, n, q+n)$ of exactly $\dot{q}+n$ dimension, except the spheres $S^{1}, S^{3}$ and $S^{7}$ (at 2).

[The case $q=1$.] By the proof of Lemma 6.4, $E$ has the homotopy type of either a product of spheres $S^{1} \times S^{n}$ with $n \in\{1,3,7\}$ or a (general) lens space $L^{3}(p, \ell)$.

[The case $n=q>1$.] Then $E$ has a cell structure $\left(S^{q} \vee S^{q}\right) \cup_{\beta} e^{2 q}$. Thus by Proposition 6.1, one obtains that $E$ has the homotopy type of $S^{q} \times S^{q}$ and $q$ is in $\{3,7\}$.

[The case $n=q+1>2$.] Then $E$ has a cell structure $S^{q} \cup_{p \iota_{q}} e^{q+1} \cup e^{2 q+1}$ where $p \iota_{q} \in \pi_{q}\left(S^{q}\right) \cong Z$. By Corollary 1.9 , we have that $(q, n)=(3,4)$ and $E \simeq S^{7}$ (at 2).

[The case $2 q>n>q+1>2$.] Then $E$ has the cell structure $S^{q} \cup_{\alpha} e^{n} \cup_{\beta} e^{n+q}$. By assumption, $n<2 q$ and $\alpha$ is a suspended element, that is, $Q=S^{q} \cup_{\alpha} e^{n}$ is desuspendable. There is a map $\mu: Q \times Q \rightarrow E$ since $E$ is a stable GW-space. By Corollary 1.9 , one can construct a space $Q(2)$ satisfying

$$
H^{+}(Q(2) ; Z / 2 Z)=Z / 2^{[3]}\left[v_{q+1}, v_{n+1}\right] .
$$

From Proposition 1.7 and Corollary 1.9 , it follows that $(q, n)=(3,5)$ and $S q^{\overline{2}} v_{4}=v_{6}$. Thus $H^{+}(E ; Z / 2 Z) \cong H^{*}(S U(3) ; Z / 2 Z)$ as algebrae over the mod 2 Steenrod algebra. This implies that the 5-skeleton of $E$ has the homotopy type of $\Sigma C P^{2}$. Thus $\beta$ lies in $\pi_{7}\left(\Sigma C P^{2}\right) \cong Z$, whose generator is given by the attaching map of the 8-cell of $S U(3)$. Since $E$ is a Poincaré complex, $\beta$ has to be a generator and hence $E$ has the homotopy type of $S U(3)$.

[The case $n=2 q>2$.] Then $E$ has a cell structure $S^{q} \cup_{\alpha} e^{2 q} \cup_{\beta} e^{3 q}, q \geq 2$. Thus $H^{+}(E ; Z) \cong Z\left\{x_{q}, x_{2 q}, x_{3 q}\right\}$ with $x_{3 q}=x_{q} x_{2 q}$. If $x_{q}^{2}=0$, one has $H^{+}(E ; Z) \cong$ $\wedge\left(x_{q}, x_{2 q}\right)$ which contradicts Proposition $3.3(2)$. Thus $x_{q}^{2} \neq 0$ and hence $H^{+}(E$; $Q) \cong Q\left[x_{q}\right] /\left(x_{q}^{4}\right)$. Then from Proposition 2.1, we obtain that $(q, n)=(2,4)$ and

$$
H^{+}(E ; Z) \cong H^{+}\left(C P^{3} ; Z\right) \text {. }
$$

Thus the 4-skeleton of $E$ has the homotopy type of $C P^{2}$. Hence the attaching map of the top cell lies in $\pi_{5}\left(C P^{2}\right) \cong Z$, whose generator is the attaching map of 
the 6-cell of $C P^{3}$. Since $E$ is a Poincare complex, $\beta$ must be a generator in $\pi_{5}\left(C P^{2}\right)$. This implies that $E$ has the homotopy type of $C P^{3}$.

[The case $n>2 q>2$.] Then $E$ has the homotopy type of $S^{q} \cup_{\alpha} e^{n} \cup e^{q+n}$ with $\alpha \in \pi_{n-1}\left(S^{q}\right)$. Since $x_{q}^{2}=0$, one has $H^{+}(E ; Z) \cong \wedge\left(x_{q}, x_{n}\right)$ where both $q$ and $n$ are odd by Proposition 3.3 (2). Then from Theorem 4.2 and Propositions 5.1 and 5.2 it follows that $(q, n)=(3,7)$. Hence we obtain

$$
H^{+}(E ; Z / 2 Z) \cong \wedge\left(\bar{x}_{q}, \bar{x}_{n}\right)
$$

with the trivial action of the mod 2 Steenrod algebra. Since $(q, n)=(3,7)$, the attaching element $\alpha$ of the 7-cell in $E$ is of the form $\alpha=k \omega$, where $\omega$ is the Blakers-Massey element in $\pi_{6}\left(S^{3}\right) \cong Z / 12 Z$. We have that $k$ is odd or $k \equiv 0 \bmod$ 4. In fact, if $\lambda \equiv 2 \bmod 4, \alpha$ is desuspendable at 2 and so is the space $Q=\left(S^{3} \cup_{\alpha}\right.$ $\left.e^{7}\right)_{(2)}$.

Then one can construct a space $Q(2)$ from which one can deduce a contradiction to the result of Sigrist-Suter [S-S] (since the result in [S-S] is essentially a result localised at 2 ).

If $\lambda$ is odd or $\lambda \equiv 0 \bmod 4$, the pull-back $E_{k \omega}$ by $k \iota_{7}$ from the principal bundle $S p(2) \rightarrow S^{7}$ is known to be an $\mathrm{H}$-space and thus it is a GW-space (see $[\mathrm{H}-\mathrm{R}]$ and $[\mathrm{Z}])$.

In case $k \equiv 0 \bmod 12$, the 6-skeleton of $E$ has the homotopy type of $S^{3} \vee S^{7}$. Thus $E$ has the same homotopy type with $S^{3} \times S^{7}$ and hence with $E_{k \omega}$.

In case $k \equiv 4$ or $8 \bmod 12$, the 6-skeleton of $E$ has the homotopy type of $S^{3} \vee S^{7}$ at 2 and of $S^{3} \cup_{\omega} e^{7}$ at odd primes.

Thus $\pi_{9}(Q)$ is isomorphic with $Z\left[\iota_{3}, \iota_{7}\right] \oplus(2$ torsion $)$ and $E$ has the homotopy type of $S^{3} \times S^{7}$ at 2 , and hence the attaching map of the top cell of $E$ is the same as that of $E_{k \omega}$ at 2 . At odd primes, $\pi_{9}(E)$ is isomorphic to $\pi_{9}(S p(2)) \cong 0$. Let us consider the homotopy fibre $F \rightarrow Q$ of the inclusion $Q \rightarrow E_{k \omega}$. By using the Serre spectral sequence associated with the above (homotopy) fibration, we deduce that $\pi_{9}(F) \cong Z$ at odd primes. Since $E_{k \omega}$ has the rational homotopy type of the product of odd spheres, $\pi_{10}\left(E_{k \omega}\right)$ is finite and hence $\pi_{9}(Q)$ is isomorphic to $Z$ at odd primes. Thus $\pi_{9}(Q)$ is isomorphic with $Z \oplus(2$ torsion $)$ in which a generator of the free part is given by the attaching map of the 10-cell of $E_{k \omega}$, and the attaching map of the top cell of $E$ is in the free part. On the other hand, $\beta$ has to be a generator, since $E$ is a Poincaré complex. Thus $E \simeq E_{k \omega}$.

In case $k$ odd, a similar argument as above shows that $\pi_{9}(Q)$ is isomorphic to $\pi_{9}\left(S^{3} \cup_{\omega} e^{7}\right) \cong Z$ and the generator is given by the attaching map of the 10-cell of $E_{k \omega}$. Thus $E \simeq E_{k \omega}$, since $E$ is a Poincaré complex.

This completes the proof of the main theorem. 


\section{Appendix}

Let $E$ and $B$ be connected CW complexes and consider a fibration

$$
F \stackrel{\iota}{\rightarrow} E \stackrel{\pi}{\rightarrow} B
$$

with fibre $F$ a (not necessarily connected) $\mathrm{CW}$ complex. It gives rise to the following two fibrations:

$$
\begin{gathered}
\Omega B \stackrel{q}{\rightarrow} F \stackrel{\iota}{\rightarrow} E, \\
\Omega E \stackrel{\Omega \pi}{\longrightarrow} \Omega B \stackrel{q}{\rightarrow} F .
\end{gathered}
$$

Now suppose that $\iota$ is null homotopic. It follows from (A.2) that $q$ has a right inverse $s: F \rightarrow \Omega B$. So the homotopy exact sequence of (A.3) splits and we obtain

$$
\pi_{+}(\Omega B) \cong \pi_{+}(\Omega E) \oplus \pi_{+}(F),
$$

where the above isomorphism is induced by the map $h=\mu^{\circ}(\Omega \pi \times s): \Omega E \times F \rightarrow$ $\Omega B$ with $\mu$ the loop addition of $\Omega B$. Thus $h$ is a homotopy equivalence, since $\Omega B$ and $\Omega E$ have the homotopy type of a CW complex. Hence we obtain

$$
h: \Omega E \times F \simeq \Omega B
$$

Thus the following hold for any space $W$ :

$$
\begin{aligned}
& 1 \rightarrow[W, \Omega E] \stackrel{\Omega \pi_{i}}{\longrightarrow}[W, \Omega B] \quad \text { as groups, } \\
& {[W, \Omega B] \cong[W, \Omega E] \times[W, F] \quad \text { as sets. }}
\end{aligned}
$$

Here let us introduce a notion of a $\mathrm{GW}$-action. A GW-action of $E$ along $\pi$ : $E \rightarrow B$ is a map

$$
v: \Sigma \Omega E \times \Sigma \Omega B \rightarrow B
$$

with axes $\Sigma \Omega E \rightarrow E \stackrel{\pi}{\rightarrow} B$ and $\Sigma \Omega B \rightarrow B$, where the map $\Sigma \Omega B \rightarrow B$ is the evaluating map.

Then we have

Theorem A.7. If $\iota$ is null-homotopic in (A.1) and if B admits a $G W$-action of $E$ along $\pi$ (see (A.6)), then the following four statements hold:

(i) $E$ is a $G W$-space and $F$ is an H-space.

(ii) If $B$ is a GW-space, then $F$ is a homotopy commutative $H$-space.

(iii) $B$ is a GW-space if and only if the Samelson product $\langle s, s\rangle$ is trivial for a right inverse $s$ of $q$.

(iv) If there is an H-map s which is a right inverse of $q$ and if $F$ is homotopy commutative, then $B$ is a GW-space and (A.4) is an H-equivalence. 
Proof. (i) By [O, Theorem 2.7], the image of $\Omega \pi_{*}$ of (A.5) is contained in the center of $[W, \Omega E] \cong[\Sigma W, E]$ for any $W$, since a map from a suspension space to a space $X$ can be decomposed through the evaluating map $\Sigma \Omega X \rightarrow X$. Furthermore $\Omega \pi_{+}$is a monomorphism by (A.5), and hence [W, $\left.\Omega E\right]$ is an abelian group for any $W$, which implies that $E$ is a GW-space by $(0.2)$. Since $F$ is a retract of a loop space $\Omega B$, it is an $\mathrm{H}$-space.

(ii) Let us define the multiplication $\bar{H}$ of $F$ by putting $\bar{H}=q^{\circ} \mu \circ(s \times s)$, where we denote by $\mu$ the loop addition of $\Omega B$. As $\mu$ is homotopy commutative, so is $\bar{\mu}$.

(iii) Suppose that $B$ is a GW-space. Since $\Sigma F$ is a suspension space, the Whitehead product $[\operatorname{ad}(s), \operatorname{ad}(s)]$ is trivial for the adjoint map ad(s): $\Sigma F \rightarrow B$ of $s$. Recall that $[\operatorname{ad}(s), \operatorname{ad}(s)]= \pm \operatorname{ad}\langle s, s\rangle$, where $\operatorname{ad}\langle s, s\rangle$ denotes the adjoint of the Samelson product of $s$. Thus we obtain $\operatorname{ad}\langle s, s\rangle=*$.

Conversely, suppose that ad $\langle s, s\rangle=*$. For simplicity we write $\mu(x, y)=x \cdot y$. Then by the homotopy associativity of $\mu$, we obtain the following homotopy:

$$
\begin{aligned}
h(x, y) \cdot h(\bar{x}, \bar{y}) & =(\Omega \pi(x) \cdot s(y)) \cdot(\Omega \pi(\bar{x}) \cdot s(\bar{y})) \\
& \simeq(\Omega \pi(x) \cdot(s(y) \cdot \Omega \pi(\bar{x}))) \cdot s(\bar{y}) .
\end{aligned}
$$

The image of $\Omega \pi_{+}$is contained in the center as is seen in (i), and so we obtain

$$
s(y) \cdot \Omega \pi(\bar{x}) \simeq \Omega \pi(\bar{x}) \cdot s(y) .
$$

Also from the homotopy commutativity of $\Omega E$ and $F$, it then follows that

$$
\begin{aligned}
h(x, y) \cdot h(\bar{x}, \bar{y}) & \simeq(\Omega \pi(x) \cdot(\Omega \pi(\bar{x}) \cdot s(y))) \cdot s(\bar{y}) \\
& \simeq(\Omega \pi(x) \cdot \Omega \pi(\bar{x})) \cdot(s(y) \cdot s(\bar{y})) .
\end{aligned}
$$

Recalling that the loop map $\Omega \pi$ is an H-map, one has

$$
\Omega \pi(x) \cdot \Omega \pi(\bar{x}) \simeq \Omega \pi(x \cdot \bar{x})
$$

where we use the same symbol ' ' to denote the loop additions of $\Omega B$ and $\Omega E$. Let us recall that $\Omega E$ is homotopy commutative by (i), and hence

$$
\Omega \pi(x \cdot \bar{x}) \simeq \Omega \pi(\bar{x} \cdot x) .
$$

Thus we obtain

$$
\Omega \pi(x) \cdot \Omega \pi(\bar{x}) \simeq \Omega \pi(\bar{x}) \cdot \Omega \pi(x) .
$$

The hypothesis $\langle s, s\rangle=*$ implies that $s(y) \cdot s(\bar{y}) \cdot s(y)^{-1} \cdot s(\bar{y})^{-1} \simeq *$. Hence it follows that

$$
s(y) \cdot s(\bar{y}) \simeq s(\bar{y}) \cdot s(y) .
$$

Summing up we get 


$$
\begin{aligned}
h(x, y) \cdot h(\bar{x}, \bar{y}) & \simeq(\Omega \pi(x) \cdot \Omega \pi(\bar{x})) \cdot(s(y) \cdot s(\bar{y})) \\
& \simeq h(\bar{x}, \bar{y}) \cdot h(x, y),
\end{aligned}
$$

that is,

$$
\mu \circ(h \times h) \simeq \mu \circ T \circ(h \times h) .
$$

Since $h$ is a homotopy equivalence in (A.4), it then follows that

$$
\mu \simeq \mu \circ T,
$$

that is, $\Omega B$ is homotopy commutative. Thus $B$ is a $\mathrm{GW}$-space.

(iv) Let $s: F \rightarrow \Omega B$ be an H-map which is a right inverse of $q$. Then the H-deviation $\mathrm{HD}(s)$ of $s$ satisfies $\mathrm{HD}(s) \simeq *$, where the $\mathrm{H}$-deviation $\mathrm{HD}(s)$ : $F \wedge F \rightarrow \Omega B$ is given by

$$
\mathrm{HD}(s)(x \wedge y)=s(x) \cdot s(y) \cdot s(x+y)^{-1}
$$

where + denotes the multiplication of $F$. It follows that

$$
\mathrm{HD}(s)(y \wedge x)=s(y) \cdot s(x) \cdot s(y+x)^{-1} .
$$

Since $F$ is homotopy commutative, we have $s(x+y) \simeq s(y+x)$. Thus we have

$$
\begin{aligned}
\mathrm{HD}(s)(x \wedge y) \cdot \mathrm{HD}(s)(y \wedge x)^{-1} & \simeq s(x) \cdot s(y) \cdot s(x+y)^{-1} \cdot s(y+x) \cdot s(x)^{-1} \cdot s(y)^{-1} \\
& \simeq s(x) \cdot s(y) \cdot s(x)^{-1} \cdot s(y)^{-1} \\
& =\langle s, s\rangle(x \wedge y) .
\end{aligned}
$$

This implies that $\langle s, s\rangle \simeq *$, and hence $B$ is a GW-space by (iii). Further, by (A.8) we have

$$
\begin{aligned}
\mu \circ(h \times h)((x, y),(\bar{x}, \bar{y}) & \simeq h(x, y) \cdot h(\bar{x}, \bar{y}) \\
& \simeq(\Omega \pi(x) \cdot \Omega \pi(\bar{x})) \cdot(s(y) \cdot s(\bar{y}))
\end{aligned}
$$

which, by using the H-structure of maps $s$ and $\Omega \pi$, changes up to homotopy into the following:

$$
\begin{aligned}
& \simeq \Omega \pi(x \cdot \bar{x}) \cdot s(y+\bar{y}) \\
& =h(x \cdot \bar{x}, y+\bar{y}) .
\end{aligned}
$$

This implies that $h$ is an $\mathrm{H}$-map and hence $\Omega B$ is $\mathrm{H}$-equivalent to $\Omega E \times F$. This completes the proof of the theorem.

Corollary A.9. (i) The standard lens space $L(p)=S^{3} /(Z / p Z)$ is a $G W$ space for all $p \geq 1$.

(ii) $C P^{3}=S^{7} / T^{1}$ is a $G W$-space.

Proof. (i) Put $F=Z / p Z, E=S^{3}$ and $B=L(p)$. They satisfy the conditions 
of Theorem A.7. So it suffices to show that $s: F \rightarrow \Omega E$ is an H-map. The Hdeviation of $s$ is in the set $[F \wedge F, \Omega E] \cong[F * F, E] \cong\left[\vee_{\alpha} S_{\alpha}^{1}, S^{3}\right] \cong \bigoplus_{\alpha} \pi_{1}\left(S^{3}\right)=0$. Hence $\operatorname{HD}(s) \simeq *$, that is, $s$ is an H-map. From (iv) of Theorem A.7, it follows that $B=L(p)$ is a $\mathrm{GW}$-space.

(ii) Put $F=T^{1}, E=S^{7}$ and $B=C P^{3}$. They satisfy the conditions of Theorem A.7, since $C P^{3}$ is a Whitehead space and $\Sigma \Omega C P^{3}$ has the homotopy type of a wedge sum of spheres. The H-deviation of $s: F \rightarrow \Omega E$ is in the set $[F \wedge F$, $\Omega E] \cong \pi_{3}\left(S^{7}\right)=0$, whence $s$ is an H-map. From (iv) of Theorem A.7, it follows that $B=C P^{3}$ is a $\mathrm{GW}$-space. This implies the corollary.

Remark. It is well-known that $\Omega S^{2}$ has the same homotopy type of $\Omega S^{3} \times$ $T^{1}$ and the latter space is homotopy commutative. If we put $F=T^{1}, E=S^{3}$ and $B=S^{2}$, they satisfy the conditions of Theorem A.7, but a splitting $s: T^{1} \rightarrow \Omega S^{2}$ cannot be an H-map. In fact, its H-deviation is the adjoint of the Hopf map $\eta$ : $S^{3} \rightarrow S^{2}$, and $S^{2}$ is not a GW-space. Thus the space $\Omega S^{2}$ has two completely different loop structure: One is homotopy commutative and the other is not.

\section{References}

[Ad1] Adams, J.F., On the non-existence of elements of the Hopf invariant one, Ann. of Math., 72 (1960), 20-104.

[Ad2] H-spaces with few cells, Topology, 1 (1961), 67-72.

[Ag] Aguadé, J., Decomposable free loop spaces, Can. J. Math., 39 (1987), 938-955.

[B-T] Browder, W., and Thomas, E., On the projective plane of an H-space. Ill. J. Math., 7 (1963), 492-502.

[H-R] Hilton, P. and Roitberg, J., On principal $S^{3}$ bundles over spheres, Ann. of Math., 90 (1969). 91-107.

[He] Hemmi, Y., The projective plane of an H-pairing, J. of Pure and Appl. Alg. (to appear).

[Hu] Hubbuck, J.R., Generalized cohomology operations and H-spaces of low rank, Trans. of A.M.S., 141 (1969), 335-360.

[I] Iwase, N., H-spaces with generating subspaces, Proc. Roy. Soc. of Edinburgh (A), 111A (1989), 199-211.

[I-Y] Iwase, N. and Yano, K., Homological image of evaluation maps, preprint.

[K] Kachi, H., Homotopy commutativity of the loop space of a finite CW-complex. Hiroshima Math. J., 20 (1990), 365-384.

[M] McGibbon, C.A., Higher forms of homotopy commutativity and finite loop spaces, Math. Z., 201 (1989), 363-374.

[O] Oda, N., Pairings and copairings in the category of topological spaces, preprint.

[S-S] Sigrist, F. and Suter, U., Eine anwendug der K-theorie in der Theorie der H-Räume, Comment. Math. Helv., 47 (1972), 36-52.

[S] Stasheff, J.D., On homotopy abelian H-spaces, Proc. Cambridge Phil. Soc., 57 (1971), $734-745$.

[Th1] Thomas, E., Steenrod squares and H-spaces I, Ann. of Math., 77 (1963), 306-317; II, Ann. of Math., 81 (1965), 473-495.

[Th2] , On the mod 2 cohomology of certain H-spaces, Comment. Math. Helv., 37 (1962), $132-140$ 
[Th3] , On functional cup products and the transgression operator, Arch. der Math., 12 (1961), 435-444.

[To] Toda, H., Composition methods in homotopy groups of spheres, Ann. of Math. Studies, 49 , Princeton University Press, Princeton, 1962.

[Z] Zabrodsky, A., The classifications of simply connected H-spaces with three cells I, Math. Scand., 30 (1972), 193-210. 